\title{
Complete classification of four-dimensional black hole and membrane solutions in IR-modified Hořava gravity
}

\author{
Carlos Argüelles, ${ }^{a, b}$ Nicolás Grandi ${ }^{c, d}$ and Mu-In Park ${ }^{e, 1}$ \\ ${ }^{a}$ ICRANet, \\ P.zza della Repubblica 10, I-65122 Pescara, Italy \\ ${ }^{b}$ Dipartimento di Fisica, Sapienza Università di Roma, \\ P.le Aldo Moro 5, I-00185 Rome, Italy \\ ${ }^{c}$ Instituto de Física de La Plata - CONICET 8 Dto. de Física, \\ Universidad Nacional de La Plata, \\ C.C. 67, 1900 La Plata, Argentina \\ ${ }^{d}$ Abdus Salam International Center for Theoretical Physics, Associate Scheme, \\ Strada Costiera 11, 34151, Trieste, Italy \\ ${ }^{e}$ Research Institute for Basic Science, Sogang University, \\ Seoul, 121-742, Korea \\ E-mail: carlos.arguelles@icranet.org, grandi@fisica.unlp.edu.ar, \\ muinpark@gmail.com
}

ABSTRACT: Hořava gravity has been proposed as a renormalizable, higher-derivative gravity without ghost problems, by considering different scaling dimensions for space and time. In the non-relativistic higher-derivative generalization of Einstein gravity, the meaning and physical properties of black hole and membrane space-times are quite different from the conventional ones. Here, we study the singularity and horizon structures of such geometries in IR-modified Hořava gravity, where the so-called "detailed balance" condition is softly broken in IR. We classify all the viable static solutions without naked singularities and study its close connection to non-singular cosmology solutions. We find that, in addition to the usual point-like singularity at $r=0$, there exists a "surface-like" curvature singularity at finite $r=r_{S}$ which is the cutting edge of the real-valued space-time. The degree of divergence of such singularities is milder than those of general relativity, and the Hawking temperature of the horizons diverges when they coincide with the singularities. As a byproduct we find that, in addition to the usual "asymptotic limit", a consistent flow of coupling constants, that we called "GR flow limit", is needed in order to recover general relativity in the IR.

KeYwords: Models of Quantum Gravity, Black Holes, Spacetime Singularities

ArXiv EPrint: 1508.04380v3

\footnotetext{
${ }^{1}$ Corresponding author.
} 


\section{Contents}

1 Introduction 1

2 The $\mathrm{D}=4$ black hole and membrane solutions in general relativity 2

2.1 Metric ansatz and general solution 3

2.2 Singularities and horizons 4

3 The $\mathrm{D}=4$ black hole and membrane solutions in IR-modified Hořava gravity $\quad 6$

3.1 IR-modified Hořava gravity and GR flow limit without fine tuning 6

3.2 Metric ansatz and general solution with IR Lorentz invariance $(\lambda=1) \quad 8$

$\begin{array}{llr}3.3 & \text { Unusual singularities and horizons } & 9\end{array}$

3.3.1 Flat membrane solution $(k=0) \quad 11$

3.3.2 Hyperbolic membrane solution $(k=-1) \quad 14$

$\begin{array}{ll}\text { 3.3.3 Spherical membrane solution }(k=+1) & 17\end{array}$

$\begin{array}{lll}4 & \text { Thermodynamics } & 19\end{array}$

5 Connection to time-dependent cosmological solutions 21

6 Concluding remarks $\quad 24$

\section{Introduction}

In 2009, Hořava proposed a renormalizable gravity theory with improved ultraviolet (UV) behavior, which reduces to Einstein gravity with a non-vanishing cosmological constant in infrared (IR). Such improved behavior is obtained at the price of abandoning Einstein's equal-footing treatment of space and time [1,2]. Since then, various aspects of the theory and its solutions have been studied [3-43]. The original Hořava model satisfying the so-called "detailed balance" condition was shown to have several problems [3]: (i) A fine-tuning dynamical mechanism is needed, in order to subtract the infinite cosmological constant arising due to the flow of the theory in the IR limit, (ii) the black hole solution in the Hořava model does not recover the usual Schwarzschild-AdS black hole, (iii) for vanishing cosmological constant, the Newtonian potential cannot be obtained in the weak field approximation. ${ }^{1}$

\footnotetext{
${ }^{1}$ We are considering only the "non-projectable" case where there is the space dependance in the lapse function $N$, as well as some possible time dependence. For the study of the Newtonian potential in the "projectable" case (or its a variant, called "covariant Hořava-Lifshitz gravity"), where there is only time dependence in $N$, see e.g. [44-47].
} 
In [4] an IR modification which contains the flat Minkowski vacuum solution has been studied, by introducing a term proportional to the Ricci scalar of the spatial geometry $\mu^{4} R^{(3)}$. This was called a "soft-breaking" of the detailed balance condition, with threedimensional Newton's constant $\sim \mu^{-1}$ [2] in the vanishing cosmological constant case. Later this was generalized to the case with an arbitrary cosmological constant such that the solutions of [3] and [4] are recovered as some particular limits by introducing the IR-modification term $\omega R^{(3)}$ with a new parameter $\omega[5]$. Actually, it turns out that this "IR-modified Hořava gravity" does not have the above-mentioned drawbacks of the original Hořava model [6].

Recently, the black "plane" solution [7], and more generally the "topological" black holes with arbitrary, constant curvature, horizons [8, 9], which includes the black hole solutions as the spherical case as well as the hyperbolic and plane membrane solutions, have been studied in the original Hořava model with detailed balance in four dimensions. In this paper, we consider the generalized model with the IR-modification term proportional to $\omega R^{(3)}$ with an arbitrary IR-modification parameter $\omega$. The resulting equations may provide the black membrane geometry without introducing matter, due to the higher spatial-derivative terms which were absent in general relativity. Here, we study the singularity and horizon structure of such space-times in IR-modified Hořava gravity and classify all the viable solutions without naked singularities. In particular, we find that there exists a surface-like curvature singularity at $r=r_{S}$ as a cutting edge of our space-time, where the real-valued space-time ends and unconventional complex-valued metric starts, as well as the usual point-like singularity at $r=0$ (for some earlier work, see [10]). We find that their degrees of divergence are milder than those of general relativity (GR), and Hawking temperatures for the black hole and membrane geometries are finite unless the singularities coincide with the outermost horizons. And also, we find that the asymptotic limit is not enough to recover the conventional results of GR but we need another limit, called the "GR flow limit", which achieves a peculiar form of flows of coupling constants.

The plan of this paper is as follows. In section II, we revisit the static black hole and membrane solutions in four-dimensional GR and we classify all the viable solutions without naked curvature singularities, in a manner which is in parallel with the reduced action approach to IR-modified Hořava gravity to be pursued later in section III. In section IV, we study the thermodynamics of the black hole and membrane geometries, and find that the Hawking temperature becomes infinity when the curvature singularity sits on the outermost horizon. In section $\mathrm{V}$, we study its close connection to the conditions for the non-singular Friedman-Lemaître-Robertson-Walker (FLRW) type cosmology. In section VI, we conclude with several remarks.

\section{The $\mathrm{D}=4$ black hole and membrane solutions in general relativity}

It is known that in four-dimensional GR with Minkowski vacuum, i.e., with vanishing cosmological constant $\Lambda=0$, the black membrane solution has a naked singularity. This situation changes when a cosmological constant is introduced, and in particular for the case of AdS vacuum $(\Lambda<0)$ a horizon which hides the singularity appears. This is basically due 
to the additional "attraction" caused by the negative cosmological constant, in contrast to "null" or "repulsion" for the cases $\Lambda \geq 0 .^{2}$

In the present section, we summarize these known results $[51]^{3}$ in the context of topological black holes, which describes the black hyperbolic membrane and plane solutions as well as the black hole solution in a unified way [53-57], in parallel with the approach to IR-modified Hořava gravity followed in the next section.

\subsection{Metric ansatz and general solution}

We start by considering the Einstein gravity action with a cosmological constant $\Lambda$ which reads $(c \equiv 1)$

$$
S_{\mathrm{EH}}=\frac{1}{16 \pi G} \int d^{4} x \sqrt{-g}(R-2 \Lambda) .
$$

We will be interested in static solutions to the above action with a maximally symmetric (i.e., constant curvature) two-dimensional slice. Then, let us consider the following metric ansatz,

$$
d s^{2}=-N^{2}(r) d t^{2}+\frac{d r^{2}}{f(r)}+r^{2} d \Omega_{k}
$$

where the sub-metric

$$
d \Omega_{k}=\left(\frac{d \rho^{2}}{1-k \rho^{2}}+\rho^{2} d \phi^{2}\right)
$$

describes the two-dimensional surface with a constant scalar curvature, $R^{(2)}=2 k$. Without loss of generality, one may take $k=+1,0,-1$ for spherical, plane, and hyperbolic geometries, respectively. For $k= \pm 1$, this can be written as the standard form in the coordinates $(\theta, \phi)$

$$
d \Omega_{k}= \begin{cases}d \theta^{2}+\sin ^{2} \theta d \phi^{2}, & (k=+1), \\ d \theta^{2}+\sinh ^{2} \theta d \phi^{2}, & (k=-1),\end{cases}
$$

by considering $\rho=\sin \theta, \sinh \theta$, respectively.

By substituting the metric ansatz into the action (2.1), the resulting reduced action, after angular integration, is given by

$$
S_{\mathrm{EH}}=\frac{\Omega_{k}}{16 \pi G} \int d t d r \frac{N}{\sqrt{f}}\left(2\left(k-f-r f^{\prime}\right)-2 \Lambda r^{2}\right),
$$

where the prime (') denotes the derivative with respect to $r$ and $\Omega_{k}$ is the volume of the two-dimensional surface with curvature $2 k$. The resulting equations of motions read

$$
-\left(k-f-r f^{\prime}\right)+\Lambda r^{2}=0, \quad\left(\frac{N}{\sqrt{f}}\right)^{\prime}=0,
$$

obtained by varying the functions $N$ and $f$, respectively. One can obtain the general solution as

$$
N^{2}=f=k-\frac{\Lambda}{3} r^{2}-\frac{2 M}{r},
$$

\footnotetext{
${ }^{2}$ This may also explain why one can have a black hole solution in three-dimensional AdS space, known as BTZ solution, but not in flat or dS space [48-50].

${ }^{3}$ For a more recent, extensive study, see [52].
} 
by setting $N / \sqrt{f}=$ constant $\equiv 1$ at the spatial infinity, $r=\infty$. Here $M$ is an integration constant, which agrees with ADM mass for the black hole $(k=+1)$ case, and generally ' $4 \pi \times$ ADM mass density' for the flat $(k=0)$ and hyperbolic $(k=-1)$ membranes.

\subsection{Singularities and horizons}

In order to make the singularities of the solution explicit, we consider the curvature invariants,

$$
\begin{aligned}
R & =4 \Lambda, \\
R^{\mu \nu \alpha \beta} R_{\mu \nu \alpha \beta} & =\frac{8}{3} \Lambda^{2}+\frac{48 M^{2}}{r^{6}},
\end{aligned}
$$

the later manifesting a curvature singularity with the power of $r^{-3}$ at $r=0$, without any $k$ dependence. This singularity needs to be hidden in our observable space-time, by forming an event horizon around, following the cosmic censorship conjecture [58]. Notice that, due to the singularity at $r=0$, we can consider the ranges of $r>0$ and $r<0$ as representing different solutions. Moreover, since the solution for $r<0$ can be mapped into that for $r>0$ by replacing $M \rightarrow-M$, we can restrict our attention to the solution for $r>0$ and consider both signs of the mass, without any loss of generality.

In order to see the horizon structure of the solution, we need to know the positive roots of the cubic polynomial obtained by multiplying $f(r)$ by $-3 r$, namely $\Lambda r^{3}-3 k r+6 M$, whose number can be also obtained by Descartes' rule of signs, as equal to the number of sign changes between consecutive nonzero coefficients, or less than it by an even number.

Let us first consider the case $k=0$, i.e., the flat membrane (figure 1 (left)). In this case, there is a horizon only if $\Lambda$ and $M$ have different sign. It is located at

$$
r_{+}=\left(\frac{-6 M}{\Lambda}\right)^{1 / 3}
$$

implying that a "sensible" or "viable" membrane solution, i.e., one in which the singularity is hidden behind a horizon at $r_{+}$, exists for $M \geq 0$ when $\Lambda<0$ (AdS space). The horizon at $r=r_{+}$hides the singularity at $r=0$ and divides the causally connected region of $N^{2}(r)=f(r)>0$ outside the horizon (in which $r$ is a space-like variable) from the region of $N^{2}(r)=f(r)<0$ inside the horizon (in which $r$ is a time-like variable), which allows us to interpret the corresponding solution as a black plane. For $M>0$, we see that $r_{+} \rightarrow \infty$ when $\Lambda \rightarrow 0_{-}$, implying that for the $\Lambda=0$ case (flat space) there is neither a horizon at finite $r$, nor a region in which the coordinate $r$ is space-like, so that this cannot be considered as a sensible solution. The case $\Lambda>0$ has no horizon neither, and then again it is not a sensible solution. On the other hand, for $M<0$, there is a horizon at $r=r_{+}$, but we will not call this a "sensible" solution since the singularity at $r=0$ is naked as seen from the region $r<r_{+}$, where $N^{2}(r)=f(r)>0$. Of course this solution could also be interpreted as a time-dependent cosmological solution with $r$ as the time coordinate, due to the "equal-footing" treatment of space and time in GR, but such interpretation will not be possible for Hořava gravity in the forthcoming sections. ${ }^{4}$ 

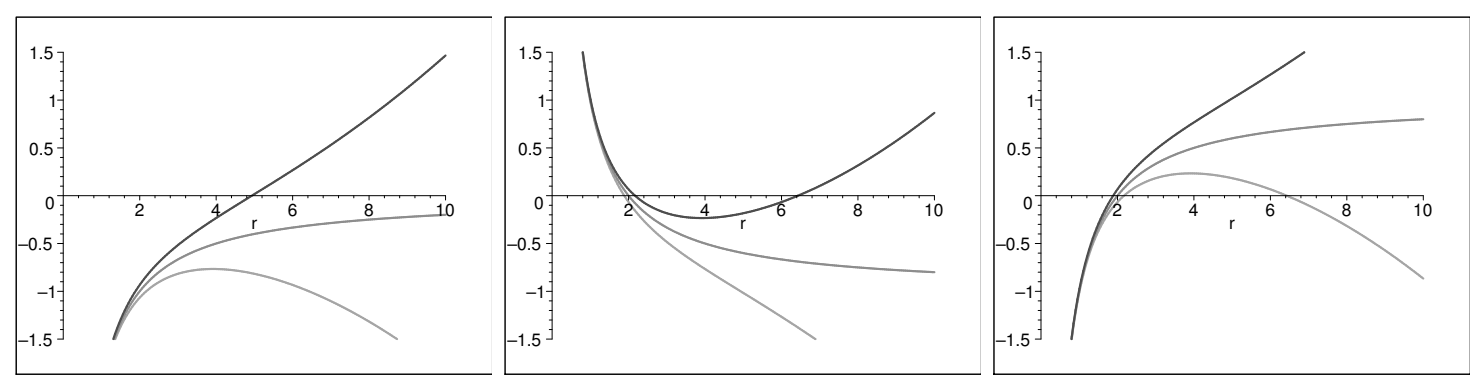

Figure 1. Plots of $f(r)$ for $k=0(M>0), k=-1(M<0)$, and $k=+1(M>0)$ from left to right. For each figure with a given $k$, the three curves denote $\operatorname{AdS}(\Lambda<0)$, flat $(\Lambda=0)$, dS $(\Lambda>0)$ spaces from top to bottom (we have plotted the AdS/dS cases for $\Lambda= \pm 0.05,|M|=1$ ). Due to the curvature singularity at $r=0$, in the case $k=0, M>0$ (left), only the AdS asymptotics (top curve) can be viable due to the existence of a horizon. The same is true for the AdS asymptotics (top curve) in the case $k=-1, M<0$ (center), where there are two horizons implying a viable black (hyperbolic) membrane solution without naked singularity. Finally, for the black hole case $k=+1, M>0$ (right), the AdS (top curve), flat (middle curve), and dS (bottom curve) solutions are all viable. All the remaining curves have a naked singularity at $r=0$ and/or no static region.
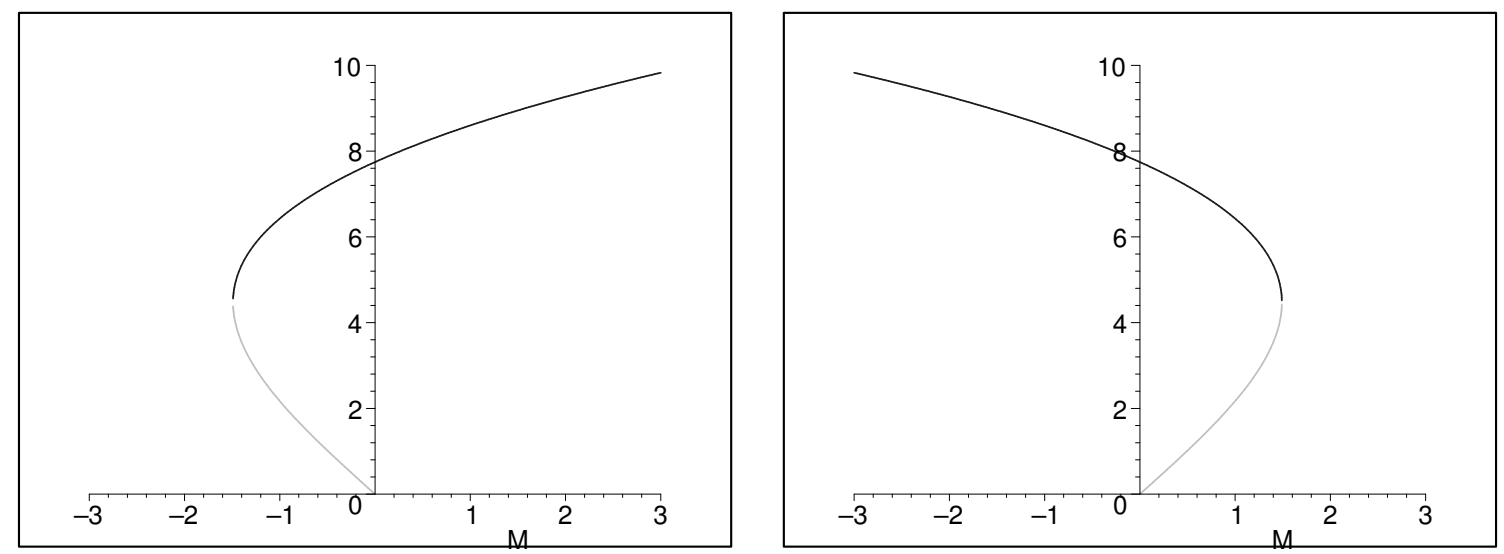

Figure 2. Plots of $r_{+}$(top curve) and $r_{-}$(bottom curve) as a function of $M$. These are the plots for $\Lambda=-0.05, k=-1$ (left) and $\Lambda=0.05, k=+1$ (right).

For the case $k=-1$, i.e., the hyperbolic membrane case, the horizon structure of the $M>0$ case is similar to the planar case: a black hole horizon exists for $\Lambda<0$ and there is no horizon for $\Lambda \geq 0 .^{5}$ The situation is quite different for the $M<0$ case (figure 1 (center) and figure 2 (left)), where a membrane solution without naked singularities is possible for $\Lambda<0$ provided $|\Lambda|^{-1}<9 M^{2}$ [59], with inner and outer black membrane horizons sitting at $r_{-}$and $r_{+}$respectively (with $r_{-}<r_{+}$)

$$
\left.r_{+}=\sqrt{\frac{3}{|\Lambda|}} \cos \left(\frac{1}{3} \arcsin \sqrt{9 M^{2}|\Lambda|}\right)-\sqrt{\frac{1}{|\Lambda|}} \sin \left(\frac{1}{3} \arcsin \sqrt{9 M^{2}|\Lambda|}\right)\right),
$$

\footnotetext{
${ }^{4}$ Actually, this case corresponds to flipping $f(r) \rightarrow-f(r)$ together with $(\Lambda, M) \rightarrow(-\Lambda,-M)$ in figure 1 (left).

${ }^{5}$ Note that this corresponds to shifting of $f(r) \rightarrow f(r)-1$ in figure 1 (left).
} 


$$
\left.r_{-}=\frac{2}{\sqrt{|\Lambda|}} \sin \left(\frac{1}{3} \arcsin \sqrt{9 M^{2}|\Lambda|}\right)\right) .
$$

On the other hand for the case $\Lambda \geq 0(M<0)$, there is a single horizon at $r_{-}$but the singularity at $r=0$ is naked in the causal region $0 \leq r<r_{-}$, as in the planar case.

Finally, the case $k=+1$, i.e., spherical horizon, is the well-known black hole solution. For $M>0$ (figure 1 (right) and figure 2 (right)), there is a single horizon for $\Lambda \leq 0$ located at

$$
r_{+}= \begin{cases}2 M & \text { for } \Lambda=0, \\ \left(-3 M+\sqrt{|\Lambda|^{-1}+9 M^{2}}\right)^{1 / 3} \Lambda^{-1 / 3}+\left(-3 M+\sqrt{|\Lambda|^{-1}+9 M^{2}}\right)^{-1 / 3} \Lambda^{-2 / 3} & \text { for } \Lambda<0,\end{cases}
$$

while for $\Lambda>0$ there are black hole and cosmological horizons at $r_{-}$and $r_{+}$respectively, as given in (2.10) provided $|\Lambda|^{-1}<9 M^{2}$.

The basic difference between the dS black hole in the last case $(k=+1, \Lambda>0, M>0)$ and the case of negative mass, black hyperbolic membrane $(k=-1, \Lambda<0, M<0)$ is that $r_{\mp}$ is its black hole/cosmological horizon for the former case, while the inner/outer black membrane horizons of the black hyperbolic membrane, without a cosmological horizon, for the latter. The case $|\Lambda|^{-1}=9 M^{2}$ is the instance that the two horizons coincide and Hawking temperature for the black hole horizon $r_{-}$, given by

$$
T_{H}=\left.\left(\frac{\hbar}{4 \pi}\right)\left(\frac{d f}{d r}\right)\right|_{r_{-}}=\left(\frac{\hbar}{2 \pi}\right)\left(-\frac{\Lambda r_{-}}{3}+\frac{M}{r_{-}^{2}}\right),
$$

vanishes and matches with that of cosmological horizon $r_{+}$, such that a thermal equilibrium is reached (Nariai solution) for the former, while (positive) Hawking temperature for the negative mass black hyperbolic membrane vanishes (extremal black brane) for the latter.

Summarizing this section, there are two possible black membrane solutions for $k=$ $0, M>0$, or $k=-1, M<0$ without naked singularities for $\Lambda<0$. However, if we consider our current universe as a dS-like space, as implied by the current accelerating expansion [60], these membrane solutions may not be quite relevant to it. If this is the case, the relevant black membrane solutions may not exist in pure Einstein gravity without matter.

\section{The $\mathrm{D}=4$ black hole and membrane solutions in IR-modified Hořava gravity}

\subsection{IR-modified Hořava gravity and GR flow limit without fine tuning}

In order to study Hořava gravity, we write the geometry in terms of its ADM decomposition

$$
d s^{2}=-N^{2} c^{2} d t^{2}+g_{i j}\left(d x^{i}+N^{i} d t\right)\left(d x^{j}+N^{j} d t\right)
$$

and the IR-modified Hořava action then reads

$$
\begin{aligned}
S= & \int d t d^{3} x \sqrt{g} N\left[\frac{2}{\kappa^{2}}\left(K_{i j} K^{i j}-\lambda K^{2}\right)-\frac{\kappa^{2}}{2 \nu^{4}} C_{i j} C^{i j}+\frac{\kappa^{2} \mu}{2 \nu^{2}} \epsilon^{i j k} R_{i \ell}^{(3)} \nabla_{j} R^{(3) \ell}{ }_{k}\right. \\
& \left.-\frac{\kappa^{2} \mu^{2}}{8} R_{i j}^{(3)} R^{(3) i j}+\frac{\kappa^{2} \mu^{2}}{8(3 \lambda-1)}\left(\frac{4 \lambda-1}{4}\left(R^{(3)}\right)^{2}-\Lambda_{W} R^{(3)}+3 \Lambda_{W}^{2}\right)+\frac{\kappa^{2} \mu^{2} \omega}{8(3 \lambda-1)} R^{(3)}\right],
\end{aligned}
$$


where

$$
K_{i j}=\frac{1}{2 N}\left(\dot{g}_{i j}-\nabla_{i} N_{j}-\nabla_{j} N_{i}\right)
$$

is the extrinsic curvature,

$$
C^{i j}=\epsilon^{i k \ell} \nabla_{k}\left(R^{(3) j}{ }_{\ell}-\frac{1}{4} R^{(3)} \delta_{\ell}^{j}\right)
$$

is the Cotton tensor, and $\kappa, \lambda, \nu, \mu, \Lambda_{W}$, and $\omega$ are coupling constants. From the higher spatial derivatives up to six orders, the theory becomes power-counting renormalizable with the dimensionless couplings $\kappa$ and $\nu$. The last term in the action represents a "soft" violation of the detailed balance condition $[2,4,5,17]$ that modifies the IR behavior without changing the improved UV behavior. Notice that, being the action non-symmetric in space and time, it is crucial that the metric (3.1) has the right signature, with time-like coordinate $t$ and space-like coordinate $x^{i}$, so that the original Hořava reasoning on renormalizability is valid. This determines $t$ as the time coordinate uniquely, in contrast to GR case.

Naively, one might expect that Hořava gravity would reduce to GR by assuming higherderivative terms are negligible at large distances, i.e., low energy, but there are some subtleties involved. For example, the truncated theory, which is effective at large distances, has a different constraint structure than that of the full theory $[1,34]$. So in order to recover GR, we consider the more general limiting procedure which entails the flow of the coupling constants as well as that of the characteristic length scale. Actually, we find that in order to recover GR, the coupling constants need to flow as

$$
\lambda \rightarrow 1, \quad \mu \rightarrow 0, \quad \nu \rightarrow \infty, \quad \Lambda_{W} \rightarrow \infty,
$$

with

$$
\mu^{2} \Lambda_{W}^{2} \sim \text { fixed, } \quad \mu^{2} \omega \sim \text { fixed. }
$$

In this flow, all the higher spatial derivative terms and the term proportional to $\mu^{2} \Lambda_{W} R^{(3)}$ vanish, and only the kinetic, cosmological constant, and IR-modification terms remain. We note that this kind of consistent flow is not possible in the original Hořava model with $\omega=0$, without introducing a hypothetical fine-tuning mechanism in order to subtract an infinite constant and get a finite cosmological term [3]. Now, by comparing with the Einstein-Hilbert action (recovering the speed of light $c$ ) [6,61],

$$
S_{\mathrm{EH}}=\frac{c^{4}}{16 \pi G} \int d t d^{3} x \sqrt{g} N\left[\frac{1}{c^{2}}\left(K_{i j} K^{i j}-K^{2}\right)+R^{(3)}-\frac{2 \Lambda}{c^{2}}\right],
$$

one can obtain the following relations for the fundamental parameters of $\mathrm{GR}^{6}{ }^{6}$

$$
c^{2}=\frac{\kappa^{4} \mu^{2} \omega}{32}, \quad G=\frac{\kappa^{2} c^{2}}{32 \pi}, \quad \Lambda=-\frac{3 \Lambda_{W}^{2} c^{2}}{2 \omega} .
$$

These relations imply that $\mu^{2} \omega>0$ and $\kappa^{2}>0$ from the physical conditions of $c^{2}>0$ and $G>0$. The AdS and dS space in Einstein gravity limit can be described, with $\Lambda_{W}^{2}>0$, by

\footnotetext{
${ }^{6}$ These relations generalize those of [4] for $\omega=8 \mu^{2}(3 \lambda-1) / \kappa^{2}$ to an arbitrary $\omega$ and non-vanishing $\Lambda_{W}$, but they differ from the original ones $[2,3,5,6]$.
} 
$\omega>0, \mu^{2}>0$ and $\omega<0, \mu^{2}<0$ respectively, which are degenerate in the flat space case $\Lambda_{W}=0$. Notice that these relations cannot be defined in the original Hor̆ava model with $\omega=0[2]$; this means that the $\omega=0$ case does not have a straightforward way to compare with our universe.

\subsection{Metric ansatz and general solution with IR Lorentz invariance $(\lambda=1)$}

Let us consider now the static and maximally symmetric solution with the metric ansatz (2.2)-(2.3). By substituting it into the action (3.2), the resulting reduced Lagrangian, after angular integration, is given by

$$
\begin{aligned}
\mathcal{L}=\frac{\kappa^{2} \mu^{2} \Omega_{k}}{8(1-3 \lambda) \frac{N}{\sqrt{f}}} & {\left[(2 \lambda-1) \frac{(f-k)^{2}}{r^{2}}-2 \lambda \frac{f-k}{r} f^{\prime}+\frac{\lambda-1}{2} f^{\prime 2}\right.} \\
& \left.-2\left(\omega-\Lambda_{W}\right)\left(k-f-r f^{\prime}\right)-3 \Lambda_{W}^{2} r^{2}\right] .
\end{aligned}
$$

The resulting equations of motion are

$$
\begin{array}{r}
(2 \lambda-1) \frac{(f-k)^{2}}{r^{2}}-2 \lambda \frac{f-k}{r} f^{\prime}+\frac{\lambda-1}{2} f^{\prime 2}-2\left(\omega-\Lambda_{W}\right)\left(k-f-r f^{\prime}\right)-3 \Lambda_{W}^{2} r^{2}=0, \\
\left(\frac{N}{\sqrt{f}}\right)^{\prime}\left((\lambda-1) f^{\prime}-2 \lambda \frac{f-k}{r}+2\left(\omega-\Lambda_{W}\right) r\right)+(\lambda-1) \frac{N}{\sqrt{f}}\left(f^{\prime \prime}-\frac{2(f-k)}{r^{2}}\right)=0,
\end{array}
$$

obtained by varying the functions $N$ and $f$, respectively.

For the $\lambda=1$ case, which reduces to the standard Einstein-Hilbert action in the IR limit (so that there is no Lorentz violation in IR), the solutions are obtained as [5]

$$
N^{2}=f=k+\left(\omega-\Lambda_{W}\right) r^{2}+\epsilon \sqrt{r\left[\omega\left(\omega-2 \Lambda_{W}\right) r^{3}+\beta\right]},
$$

where $\epsilon= \pm 1$ and $\beta$ is an integration constant. ${ }^{7}$

In the "asymptotic" region $r \gg\left[\beta / \omega\left(\omega-2 \Lambda_{W}\right)\right]^{1 / 3}$, the above solution behaves as

$$
N^{2}=f=k+\left(\omega-\Lambda_{W}+\epsilon|\omega| \sqrt{1-\frac{2 \Lambda_{W}}{\omega}}\right) r^{2}+\frac{\epsilon \beta}{2|\omega| \sqrt{1-2 \Lambda_{W} / \omega}} \frac{1}{r}+\mathcal{O}\left(r^{-4}\right),
$$

but, as we see, this is not enough to get the conventional results of GR. Now then, by defining a new parameter $M$ as $\beta=4 \omega M$ and considering the "GR" limit ${ }^{8}|\omega| \gg\left|\Lambda_{W}\right|$, this becomes

$$
N^{2}=f=k-\frac{\Lambda}{3 c^{2}} r^{2}-\frac{2 M}{r}+\mathcal{O}\left(r^{-4}\right),
$$

in agreement ${ }^{9}$ with the standard Schwarzschild-AdS/dS black hole (2.7), by taking $\epsilon=-1$ for the AdS/flat case $(\Lambda \leq 0$ or equivalently $\omega>0)$, and $\epsilon=+1$ for the dS case $(\Lambda>0$

\footnotetext{
${ }^{7}$ If one adds another IR-modification term $\kappa^{2} \mu^{2}(8(3 \lambda-1))^{-1} \hat{\beta} \Lambda_{W}^{2}$ as in $[2,17]$, the solution becomes $N^{2}=f=k+\left(\omega-\Lambda_{W}\right) r^{2}+\epsilon \sqrt{r\left[\left\{\omega\left(\omega-2 \Lambda_{W}\right)+\hat{\beta} \Lambda_{W}^{2} / 3\right\} r^{3}+\beta\right]}$. This can be obtained by redefining the parameters $\Lambda_{W} \rightarrow \sqrt{1-\hat{\beta} / 3} \Lambda_{W}, \omega \rightarrow \omega+(\sqrt{1-\hat{\beta} / 3}-1) \Lambda_{W}$ in (3.10). This is also true at the action level so that the IR-modification term in (3.2) is more or less unique.

${ }^{8}$ From (3.6), one obtains $|\omega| \sim\left|\Lambda_{W}^{2}\right| \gg\left|\Lambda_{W}\right|$ as $\left|\Lambda_{W}\right| \rightarrow \infty$.

${ }^{9}$ There were some, unexplained, factor disagreements in the GR limit of (3.11) with SchwarzschildAdS/dS black hole for the original definition [2, 3, 5]. Now with the new definitions of (3.7), this problem does not occur and we have perfect agreement up to order $r^{-4}$.
} 
or equivalently $\omega<0)$. Since we are interested in solutions to IR-modified Hořava gravity that flow into GR solutions under the IR limit, hereafter we only consider the $\epsilon=-1(+1)$ branch for $\omega>0(<0)$ which we call the "AdS $(\mathrm{dS})$ branch". This shows the importance of the GR flow, which achieves a peculiar form of flows of coupling constants as in (3.5)-(3.7), as well as the asymptotic limit in order to recover the results of GR. In other words:

"The GR limit of the Horava black hole/membrane is not reached in the asymptotic region generically, but only in the $\Lambda_{W}=0$ case".

This may explain the significant difference between the Schwarzschild-AdS/dS solution and the Lü, Mei, Pope's solution [3] of the original Hořava gravity with the detailed balance condition. For the latter, the GR limit cannot be defined in the asymptotic region due to the absence of $\omega$, implying that there is no way to compare to our universe.

\subsection{Unusual singularities and horizons}

The solution (3.10) has a spatial curvature invariant

$$
R^{(3)}=-6\left(\left(\omega-\Lambda_{W}\right)+\epsilon \frac{\omega\left(\omega-2 \Lambda_{W}\right) r^{3}+\beta / 2}{r \sqrt{r\left[\omega\left(\omega-2 \Lambda_{W}\right) r^{3}+\beta\right]}}\right) .
$$

This shows that, in the asymptotic limit, the solution behaves as a constant curvature space $R^{(3)} \approx-6\left[\left(\omega-\Lambda_{W}\right)+\epsilon \sqrt{\omega\left(\omega-2 \Lambda_{W}\right)}\right]$ flowing into an asymptotically AdS/dS space-time $R^{(3)} \approx 2 \Lambda$ in the GR limit (3.7).

For $\beta>0$, the usual point-like curvature singularity at $r=0$ is present as in Einstein gravity, but now with a milder form $R_{i j}^{(3)} R^{(3) i j} \approx(27 / 8) \beta r^{-3}, R_{i j k l}^{(3)} R^{(3) i j k l}=4 R_{i j}^{(3)} R^{(3) i j}-$ $R^{(3)^{2}} \approx 9 \beta r^{-3}$ in contrast to the $r^{-6}$ of the GR case (2.8). On the other hand, when $\omega\left(2 \Lambda_{W}-\omega\right) \neq 0$ and $\beta \neq 0$, the above expression shows an unusual surface-like curvature singularity, ${ }^{10}$ sitting at

$$
r_{S}=\left(\frac{\beta}{\omega\left(2 \Lambda_{W}-\omega\right)}\right)^{1 / 3}
$$

where the denominator of the second term in (3.13) vanishes and $R^{(3)} \approx \sqrt{3} \epsilon \beta^{1 / 3}\left[\omega\left(2 \Lambda_{W}-\right.\right.$ $\omega)]^{1 / 6}\left(r-r_{S}\right)^{-1 / 2}$ near $r=r_{S}$, with a lower degree of divergence than the aforementioned point-like singularity. For the case $\omega\left(2 \Lambda_{W}-\omega\right)=0$, only the point singularity at $r=$ 0 survives, with no additional singularity in the curvature invariants. Note that these singularities are physical ones which cannot be removed by coordinate transformations in the group of foliation preserving diffeomorphism.

\footnotetext{
${ }^{10}$ A similar surface singularity at $r_{\Lambda} \equiv\left(-2 M / \Lambda_{W}\right)^{1 / 3}$ has been found for the projectable form of the AdSSchwarzschild black hole solution $\left(\Lambda_{W}<0\right)[3], d s^{2}=-d t^{2}+\left(d r+\sqrt{M / r+\Lambda_{W} r^{2} / 2} d t\right)^{2}+r^{2} d \Omega$ with the detailed balance (i.e., $\omega=0$ in our context) [10]. In contrast to our non-projectable solutions, the projectable solution does not have contributions from higher-spacial derivatives due to "flat"-spatial metric $g_{i j}$, i.e., $R^{(3)}=0$, and the singularities are captured by the extrinsic curvature scalar $K$, instead. In order to compare $r_{\Lambda}$ with $r_{S}$ in our case, one might try to consider $\omega=0$ with $\beta=4 \omega M$ and obtains $r_{S}=\left(2 M / \Lambda_{W}\right)^{1 / 3}$, which disagrees with $r_{\Lambda}$. But in this case, $R^{(3)}$ in (3.13) is subtle in identifying the singularity at $r_{S}$ so that the direct connection between the singularities for the two distinct solutions is not quite manifest.
} 
In what follows, due to the singularity at $r=0$, we consider the range $r>0$ without loss of generality, as in the previous section for the GR case. Moreover, since the surface-like singularity sits exactly at the location where the square-root term in (3.10) vanishes, the metric becomes complex-valued beyond $r_{S}$. This implies that we must consider the ranges

$$
\begin{cases}r>r_{S}>0 & \text { for } \beta<0, \omega\left(2 \Lambda_{W}-\omega\right)<0, \\ 0<r<r_{S} & \text { for } \beta>0, \omega\left(2 \Lambda_{W}-\omega\right)>0\end{cases}
$$

in order to have a real-valued metric.

From the metric ansatz (2.2), we define the "observer region" of our solution as that where $t$ is the time and $r$ is the space, in other words, $N^{2}(r), f(r)>0$ so that measurements can be made by a fixed observer. Note that only in this region the power-counting renormalizable Hořava theory is correctly defined. ${ }^{11}$ So, from the point of the observer region, the singularities should be avoided or hidden behind horizons. ${ }^{12}$

Now, in order to find the horizons, we note that the horizon condition $N^{2}=f=0$ can be rewritten as $k+\left(\omega-\Lambda_{W}\right) r^{2}=-\epsilon \sqrt{r\left[\omega\left(\omega-2 \Lambda_{W}\right) r^{3}+\beta\right]}$. By squaring both sides, we need to solve the polynomial $\Lambda_{W}^{2} r^{4}+2 k\left(\omega-\Lambda_{W}\right) r^{2}-\beta r+k^{2}$ to obtain its positive zeros, and then filtering them with the additional requirement that the sign of $k+\left(\omega-\Lambda_{W}\right) r^{2}$ at the zero has to be $-\epsilon$. In this way, one can obtain the two roots, generically

$$
r_{ \pm}=S \pm \frac{1}{\left|\Lambda_{W}\right|} \sqrt{\frac{\beta}{4 S}-S^{2} \Lambda_{W}^{2}-k\left(\omega-\Lambda_{W}\right)}
$$

where

$$
\begin{aligned}
S & =\sqrt{-\frac{k\left(\omega-\Lambda_{W}\right)}{3 \Lambda_{W}^{2}}+\frac{1}{12 \Lambda_{W}^{2}}\left(Q+\frac{\Delta_{0}}{Q}\right)}, & Q & =\left(\frac{\Delta_{1}+\sqrt{\Delta_{1}^{2}-4 \Delta_{0}^{3}}}{2}\right)^{\frac{1}{3}} \\
\Delta_{0} & =4 k^{2}\left(\omega^{2}-2 \omega \Lambda_{W}+4 \Lambda_{W}^{2}\right), & \Delta_{1} & =16 k^{3}\left(\omega-\Lambda_{W}\right)^{3}+27 \Lambda_{W}^{2} \beta^{2}-144 \Lambda_{W}^{2} k\left(\omega-\Lambda_{W}\right) .
\end{aligned}
$$

Notice that the roots $r_{ \pm}$above are not necessarily positive nor real, so they may not represent real horizons for some range of parameters. We will explore this issue in the forthcoming sections. The number of horizons at positive $r$ can also be obtained by making use of Descartes' rule of signs in the aforementioned polynomial.

\footnotetext{
${ }^{11}$ In the regions of our solutions where $N^{2}(r), f(r)<0$, the signature of the metric is such that $r$ becomes the time variable. Since our system has higher $r$ derivatives, Ostrogradsky ghost might appear there, and the solution become unstable. A definitive answer would require a deeper investigation that we plan to pursue somewhere else [62]. For the time being, the above defined observer regions of our solutions can be regarded as building blocks to construct wormhole-like metrics, like those of [39-41], in which $N^{2}(r), f(r)>0$ everywhere.

${ }^{12}$ The notion of the horizon is an emergent concept at low energy and so the cosmic censorship may be violated at higher energies. But here, we adopt the cosmic censorship as an emergent notion at low energy also. We will discuss more about this point in section VI. and in a forthcoming publication [62].
} 
The roots $r_{ \pm}$may coincide at $r=r_{*}$ when there is a double root, i.e., $f\left(r_{*}\right)=f^{\prime}\left(r_{*}\right)=$ 0 , which can be solved for $\beta$ obtaining

$$
\begin{aligned}
\beta_{*}= & \frac{4}{3 \sqrt{3}\left|\Lambda_{W}\right|} \sqrt{-k\left(\omega-\Lambda_{W}\right)+|k| \sqrt{\left(\omega^{2}-2 \omega \Lambda_{W}+4 \Lambda_{W}^{2}\right)}} \\
& \times\left(2 k\left(\omega-\Lambda_{W}\right)+|k| \sqrt{\left(\omega^{2}-2 \omega \Lambda_{W}+4 \Lambda_{W}^{2}\right)}\right),
\end{aligned}
$$

as the minimum value of the integration constant $\beta$, for a given $\Lambda_{W}$. The reason of a minimum value $\beta_{*}$ can also be understood from the black hole/membrane thermodynamics (see section IV). We can also solve for $r_{*}$ to get

$$
r_{*}=\sqrt{\frac{-k\left(\omega-\Lambda_{W}\right)+|k| \sqrt{\omega^{2}-2 \omega \Lambda_{W}+4 \Lambda_{W}^{2}}}{3 \Lambda_{W}^{2}}} .
$$

On the other hand, the horizons at $r_{+}$or $r_{-}$may coincide with the surface-like singularity at $\tilde{r}_{ \pm}$when $\beta=\tilde{\beta}$ with

$$
\tilde{r}_{ \pm}=\sqrt{\frac{k}{\Lambda_{W}-\omega}}, \quad \tilde{\beta}=\omega\left(2 \Lambda_{W}-\omega\right)\left(\frac{k}{\Lambda_{W}-\omega}\right)^{3 / 2},
$$

which can be obtained, by solving $r_{S}=r_{ \pm} \equiv \tilde{r}_{ \pm}$.

\subsubsection{Flat membrane solution $(k=0)$}

We first consider the flat membrane case $k=0$ and classify the solutions according to the sign of $\beta$.

A. Case $\boldsymbol{\beta}>\mathbf{0 :}$ in this case, there is an inner horizon, for any finite $\omega$, at

$$
r_{-}=0
$$

so that the point singularity at $r=0$ is not naked unless we consider the trivial case of Minkowski vacuum, $\beta=\Lambda_{W}=0$. This is a genuine effect of the higher-derivative terms in Hořava gravity which is absent in the GR. Moreover, there is an outer horizon at

$$
r_{+}=\left(\frac{\beta}{\Lambda_{W}^{2}}\right)^{1 / 3}
$$

which exists in the AdS branch $(\epsilon=-1)$ of the solution (3.10) for $\omega>\Lambda_{W}$ and in the dS branch $(\epsilon=+1)$ for $\omega<\Lambda_{W}$. Interestingly, this outer horizon is exactly the same as (2.9) in Einstein gravity, with the identification of $\beta=4 \omega M$ and $\Lambda$ as in (3.7) and the solution is similarly interpreted as a black plane.

Now, in order to see whether the surface-like curvature singularity (3.14) is naked in our observer region or not, one might try to consider the condition for $r_{+} \geq r_{S}$ if $r_{+}$and $r_{S}$ exist. Supposing that $r_{+} \geq r_{S}$ implies $\Lambda_{W}^{2}-\omega\left(2 \Lambda_{W}-\omega\right)=\left(\Lambda_{W}-\omega\right)^{2} \leq 0$, but this is impossible if $\Lambda_{W} \neq \omega$, and the case $\Lambda_{W}=\omega$ is when $r_{+}=r_{S}$. This proves that 
$r_{+} \leq r_{S}$ if $r_{S}$ exists. According to (3.15), the surface-like singularity exists for $\beta>0$ when $\omega\left(2 \Lambda_{W}-\omega\right)>0$, and in such case the allowed region for the radial coordinate is $0<r<r_{S}$. So we can have viable black membrane solutions without naked singularities either when $r_{S}$ does not exist, i.e., $\omega\left(2 \Lambda_{W}-\omega\right) \leq 0$ or when $r_{S}$ is hidden behind the cosmological horizon $r_{+} \leq r_{S}$. Then the possible solutions are (a) $\omega=0$, (b) $\omega=2 \Lambda_{W}$, (c) $\omega>0, \omega>2 \Lambda_{W}$, (d) $\omega<0, \omega<2 \Lambda_{W}$, and (e) $2 \Lambda_{W}<\omega \leq \Lambda_{W}<0$. Some more details are in order.

(a) $\omega=0$ : this case corresponds to the plane solution in the original Hořava theory, where the GR is not recovered under the IR flows $(3.5)-(3.7)[7,8]$. Here, the surface-like singularity at $r=r_{S}$ does not exist, though the horizon at $r_{-}$does. The horizon at $r_{+}$exists for $\Lambda_{W}>0$ in the dS branch $(\epsilon=+1)$ as a cosmological horizon, with the observer region $N^{2}, f>0$ for $0<r<r_{+}$, and for $\Lambda_{W}<0$ in the AdS branch $(\epsilon=-1)$ as a black plane horizon, with the observer region for $r>r_{+}$(figure 3). However, for the case $\Lambda_{W}=0$, there is no a priori reason to choose which of the given two branches, due to the lack of a GR limit.

(b) $\omega=2 \Lambda_{W}$ : this case corresponds to $-\Lambda_{W} \rightarrow \Lambda_{W}$ in the result of (a) and so all the properties can be understood just by flipping the sign of $\Lambda_{W}$ in figure 3 .

(c) $\omega>0, \omega>2 \Lambda_{W}, \epsilon=-1$ : in contrast to the cases of (a) and (b), this case reduces to GR under the IR flows (3.5)-(3.7). But, similar to the cases of (a) and (b), the surfacelike curvature singularity at $r_{S}$ does not exist, and the $r=0$ singularity is hidden by the coincident inner horizon at $r_{-}=0$ as well as by the outer horizon at $r_{+}$, with the observer region for $r>r_{+}$(figure 4 (left)). According to the IR limit (3.7) with the identification of $\beta=4 \omega M$ as in (3.12), this case flows to the case $M>0, \Lambda<0$ of GR. However, for the case $\Lambda_{W}=0$, there is no observer region with the space-like coordinate $r$.

(d) $\omega<0, \omega<2 \Lambda_{W}, \epsilon=+1$ : as in the case (c), this case reduces to GR under the IR flows (3.5)-(3.7). Again, this case does not confront the surface-like curvature singularity at $r_{S}$. The horizons at $r_{ \pm}$exist with the observer region $0<r<r_{+}$and the horizon at $r_{+}$being a cosmological one (figure 4 (right)). According to the IR limit (3.7) with $\beta=4 \omega M$ as in (3.12), this case flows into the case $M<0, \Lambda>0$ of GR. Note that the existence of this viable solution is basically due to the existence of an inner horizon $r_{-}=0$ as a higher-spatial derivative effect that is absent in GR.

Except in the above four cases, one can confront the curvature singularity at $r_{S}$ (figure 5), but there is one interesting viable case.

(e) $2 \Lambda_{W}<\omega \leq \Lambda_{W}<0, \epsilon=+1$ : here, there is a cosmological horizon at $r_{+}$and so the curvature singularity at $r_{S}$ is always beyond the horizon $r_{+} \leq r_{S}$, as has been proven above, and hidden from the observer region $0<r<r_{+}$(figure 5 (right); middle and bottom curves).

B. Case $\boldsymbol{\beta}<\mathbf{0}$ : for $\beta<0$, there are no horizons and so the surface curvature singularity at $r_{S}$ may be naked if it exists. In such case, the point-like singularity at $r=0$ can be 

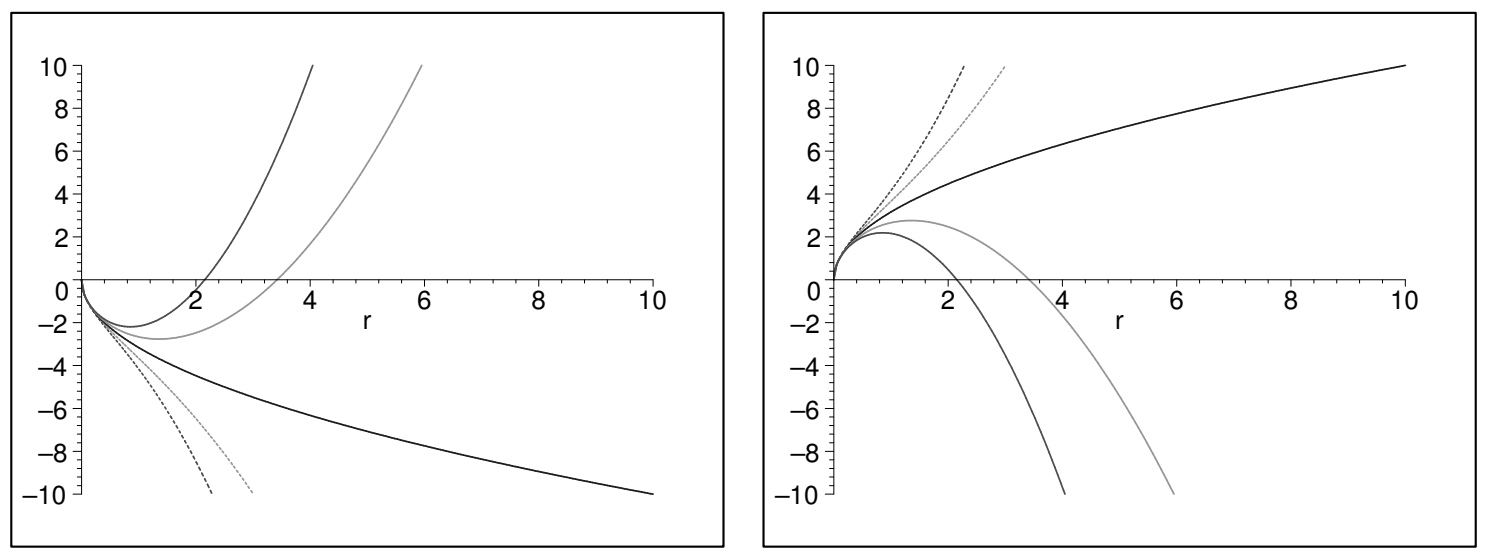

Figure 3. Plots of $f(r)$ for $k=0, \beta>0, \omega=0$. Left: case $\epsilon=-1$. We have plotted for $\Lambda_{W}=-1,-0.5,0,0.5,1(\beta=10)$ from top to bottom, respectively. The cases $\Lambda_{W}<0$ correspond to the black planes in AdS space with the horizon at $r_{-}=0$ and $r_{+}=\left(\beta / \Lambda_{W}^{2}\right)^{(1 / 3)}$. The cases $\Lambda_{W} \geq 0$ have a horizon at $r_{-}=0$ so that the singularity is not naked, but they have no region in which the variable $r$ is space-like. Right: case $\epsilon=+1$. We have plotted for $\Lambda_{W}=1,0.5,0,-0.5,-1(\beta=10)$ from bottom to top, respectively. The cases $\Lambda_{W}>0$ show the cosmological horizon in dS space at $r_{+}=\left(\beta / \Lambda_{W}^{2}\right)^{(1 / 3)}$ as well as the black plane horizon at $r_{-}=0$ so that the $r=0$ singularity is not naked. The cases $\Lambda_{W} \leq 0$ have a horizon at $r_{-}=0$ so that singularity is not naked, and the variable $r$ has the space-like signature in its whole range of $r>0$.
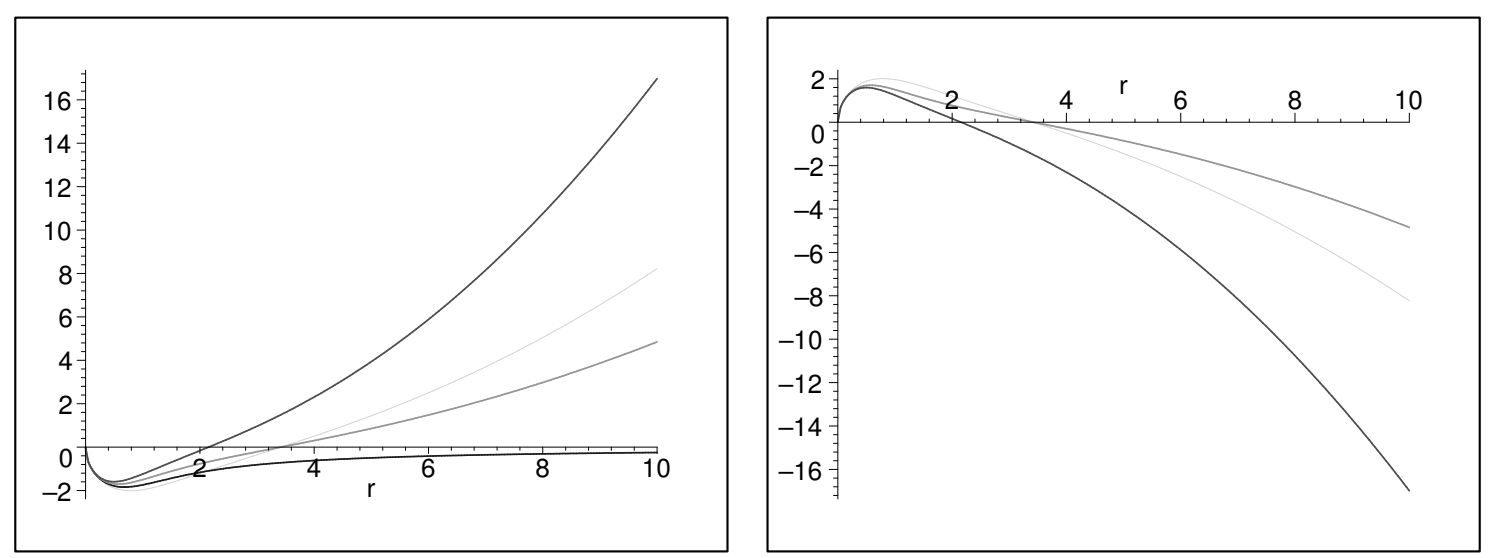

Figure 4. Plots of $f(r)$ for $k=0, \beta>0, \omega\left(2 \Lambda_{W}-\omega\right)<0$. Left: case $\epsilon=-1 \omega>0, \omega>2 \Lambda_{W}$. The three (thick) curves denote black membranes for $\Lambda_{W}=-1,-0.5,0, \omega=2(\beta=10)$ from top to bottom, respectively. The behaviors are qualitatively the same as the case (a) with $\Lambda_{W}<0$. This property extends also to $\Lambda_{W}>0$ region, if $\omega>2 \Lambda_{W}$ is satisfied, as plotted by thin curve for $\Lambda_{W}=0.5$. Right: case $\epsilon=+1, \omega<0, \omega<2 \Lambda_{W}$. The two (thick) curves denote black membranes for $\Lambda_{W}=1, \overline{0.5, \omega}=-2$ from top to bottom, respectively. The behaviors are qualitatively the same as the case (b) with $\Lambda_{W}>0$. This property extends also to $\Lambda_{W}<0$ region, if $\omega<2 \Lambda_{W}$ is satisfied, as plotted by thin curve for $\Lambda_{W}=-0.5$. 

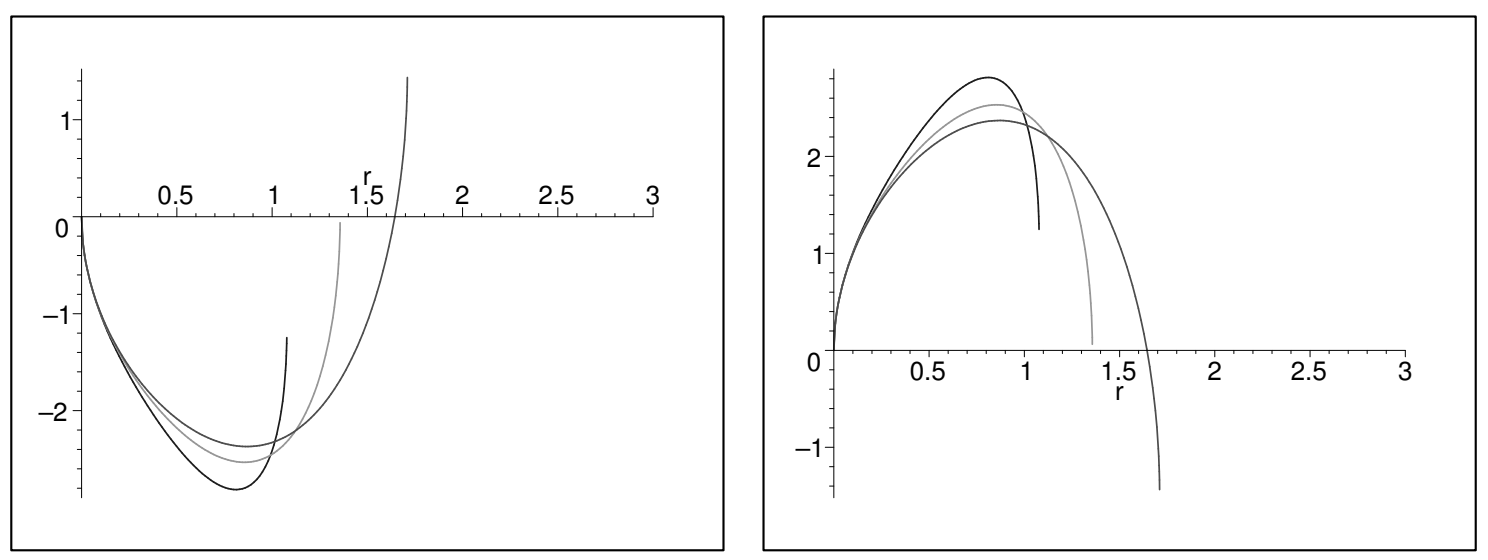

Figure 5. Plots of $f(r)$ for $k=0, \beta>0, \omega\left(2 \Lambda_{W}-\omega\right)>0$. Left: case $\epsilon=-1,0<\omega<2 \Lambda_{W}$. The three curves denote black membranes for $\Lambda_{W}=3,2,1.5, \omega=2(\beta=10)$ from bottom to top, respectively. For $\omega<\Lambda_{W}$, there is a curvature singularity at $r_{S}$ where the curve ends and beyond which it becomes complex valued, but no black hole horizon $r_{+}$(bottom curve). On the other hand, for $\omega=\Lambda_{W}$ (middle curve) and $\omega>\Lambda_{W}$ (top curve), $r_{S}$ is located on or outside the horizon $r_{+}$. Right: case $\epsilon=+1,2 \Lambda_{W}<\omega<0$. The curves denote black membranes for $\Lambda_{W}=-3,-2,-\overline{1.5, \omega}=-2(\beta=10)$ from top to bottom, respectively. For $\omega>\Lambda_{W}$, there is a curvature singularity at $r_{S}$ where the curve ends and beyond which it becomes complex valued, but no cosmological horizon $r_{+}$(top curve). On the other hand, for $\omega=\Lambda_{W}$ (middle curve) and $\omega<\Lambda_{W}$ (bottom curve), $r_{S}$ is located on or behind the cosmological horizon $r_{+}$.

excluded since the allowed region in which the metric is real, according to (3.15), is $r>r_{S}$. The surface-like singularity exists when $\omega\left(2 \Lambda_{W}-\omega\right)<0$, i.e., $\omega>0, \omega>2 \Lambda_{W}$ (figure 6 (left)) or $\omega<0, \omega<2 \Lambda_{W}$ (figure 6 (right)). In these cases, the GR limit can be taken and according to (3.7) with $\beta=4 \omega M$, they run into the GR solutions with $M<0, \Lambda<0$ and $M>0, \Lambda>0$, respectively, while $r_{S} \rightarrow 0$ matching the naked curvature singularity of those GR solutions at the origin. For other than these two cases, i.e., $2 \Lambda_{W}<\omega<0$ or $0<\omega<2 \Lambda_{W}$, there is neither real-valued metric for the whole region, nor the GR limit.

\subsubsection{Hyperbolic membrane solution $(k=-1)$}

The case $k=-1$ corresponds to the hyperbolic membrane. Its horizon structure can be understood as the intersections of the curves in figures 3 to 6 with an horizontal line at $f(r)=1$. Again, we classify the solutions according to the sign of $\beta$.

A. Case $\boldsymbol{\beta}>\mathbf{0}$ : in this case, there is no inner horizon for the AdS branch $(\epsilon=-1),{ }^{13}$ but otherwise the situation is more or less the same as the $k=0$ case. According to the same classification as before, we have the following viable cases.

(a) $\omega=0$ : here, the surface-like singularity at $r=r_{S}$ does not exist and there is no inner horizon for the AdS branch $(\epsilon=-1)$. The point-like singularity at the origin is hidden by a black membrane horizon at $r_{+}$for $\Lambda_{W}<0$, with the observer region $r>r_{+}$.

\footnotetext{
${ }^{13}$ In this case, the root formula for $r_{-}$in (3.16) does not apply since it represents the horizon for the un-physical branch in which the GR result (3.12) is not recovered.
} 

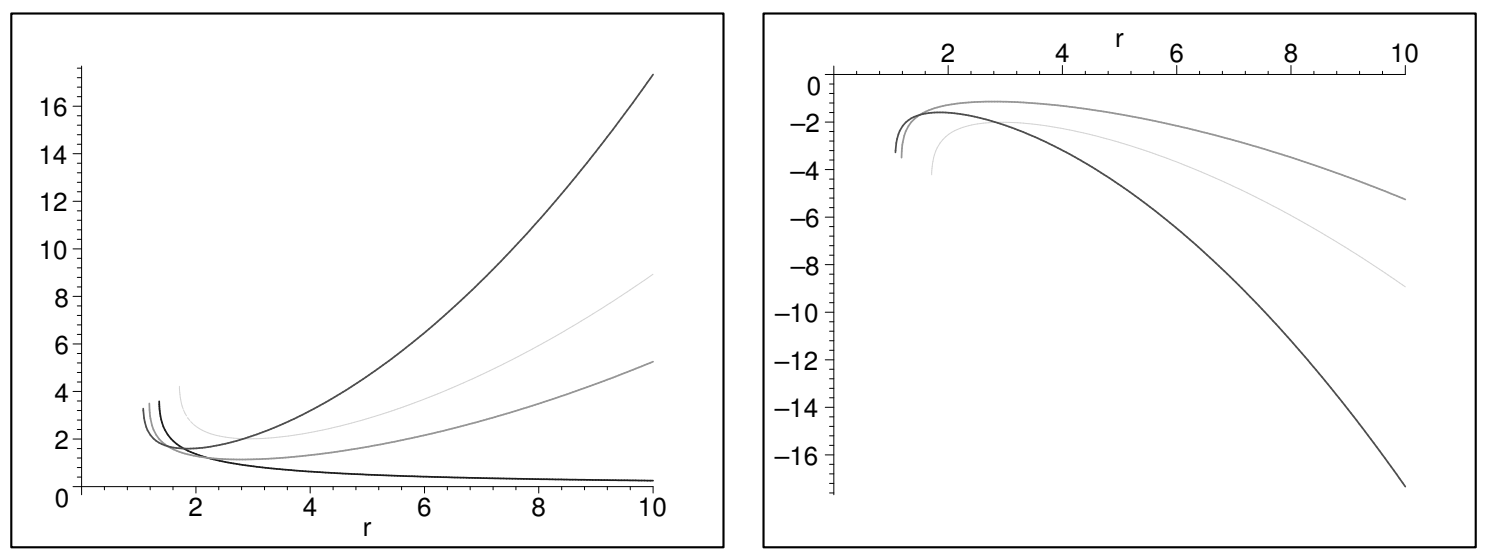

Figure 6. Plots of $f(r)$ for $k=0, \beta<0, \omega\left(2 \Lambda_{W}-\omega\right)<0$. Left: case $\epsilon=-1, \omega>0, \omega>2 \Lambda_{W}$. The curves denote black membranes for $\Lambda_{W}=-1,-0.5,0,0.5, \omega=2(\beta=-10)$ from left to right, respectively. There is a curvature singularity at $r_{S}$ where the curve ends and beyond which it becomes complex valued but without horizons. Right: case $\epsilon=+1, \omega<0, \omega<2 \Lambda_{W}$. The curves denote black membranes for $\Lambda_{W}=1,0.5,-0.5, \overline{\omega=-2}(\beta=-10)$ from left to right, respectively. There is a curvature singularity at $r_{S}$ where the curve ends and beyond which it becomes complex valued but without horizons.

On the other hand, for the dS branch $(\epsilon=+1)$, there is a black membrane horizon at $r_{-}$and a cosmological horizon at $r_{+}$with the observer region $r_{-}<r<r_{+}$for $\Lambda_{W}>0, \beta>\beta_{*}$. The two horizons meet at $r_{+}=r_{-} \equiv r_{*}$ when $\beta=\beta_{*}$. The case $\Lambda_{W} \leq 0$ can also provide an observer region $r_{-}<r$ with the black membrane horizon $r_{-}$only (figure 3 ).

(b) $\omega=2 \Lambda_{W}$ : this case corresponds to " $-\Lambda_{W}$ " $\rightarrow$ " $\Lambda_{W}$ " in the result of (a) and so all the properties can be understood just by flipping the sign of $\Lambda_{W}$.

(c) $\omega>0, \omega>2 \Lambda_{W}, \epsilon=-1$ : similar to the cases (a) and (b), the surface-like curvature singularity at $r_{S}$ does not exist, and the point-like singularity at $r=0$ is hidden by the outer black membrane horizon at $r_{+}$, with the right signature of metric for the observer region $r>r_{+}$(figure 4 (left)). This case flows to the case $M>0, \Lambda<0$ of GR. For the case $\Lambda_{W}=0$, as in the flat membrane case, there is no observer region.

(d) $\omega<0, \omega<2 \Lambda_{W}, \epsilon=+1$ : again, this case does not confront the surface-like curvature singularity since $r_{S}$ does not exist. The horizons at $r_{ \pm}$exist whenever $\beta>\beta_{*}$, the one at $r_{+}$being a cosmological one, implying that the metric has the right signature in the observer region $r_{-}<r<r_{+}$. This case flows into the case $M<0, \Lambda>0$ of GR (figure 4 (right)).

Now, for the cases in which there is the surface-like curvature singularity at $r_{S}$, we can either have no horizon, or have a black membrane horizon at $r_{+}\left(\right.$case $\left.2 \Lambda_{W}>\omega>0\right)$, but then since $r_{+} \leq r_{S}$ the surface-like singularity is naked as seen from our observer region (figure 5 (left)), or have a cosmological horizon (case $2 \Lambda_{W}<\omega<0$ ), and in 

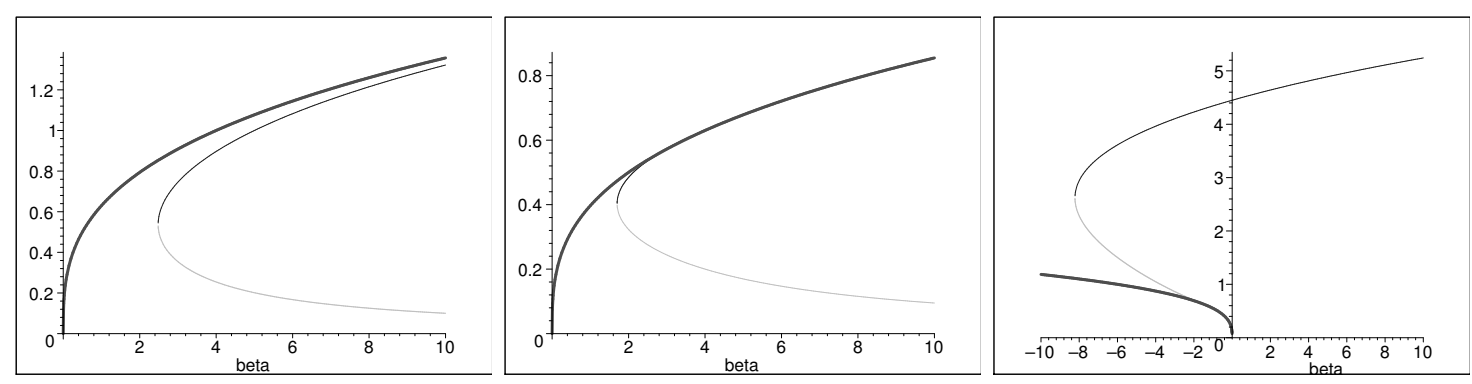

Figure 7. Plots of $r_{ \pm}$(two thin curves) and $r_{S}$ (thick curve) vs. $\beta$, for $k=-1$. Left: case $\epsilon=+1,2 \Lambda_{W}<\omega \leq \Lambda_{W}<0$, in particular for $\Lambda_{W}=-2, \omega=-2$. The plots show that $r_{+}<r_{S}$ always, independently on $\beta$ so that the surface singularity is hidden whenever the horizon exists. Center: case $\epsilon=+1,2 \Lambda_{W}<\Lambda_{W}<\omega<0$, in particular for $\Lambda_{W}=-5, \omega=-2$. The plots show that $r_{+} \leq r_{S}$, if the cosmological $r_{+}$exists, for $\beta \leq \tilde{\beta}\left(r_{S}=r_{+} \equiv \tilde{r}_{+}\right.$for $\left.\beta=\tilde{\beta}\right)$ but for $\beta>\tilde{\beta}$ there is no cosmological horizon so that the surface singularity is naked. Right: case $\epsilon=-1, \omega>0, \omega>2 \Lambda_{W}$, in particular for $\Lambda_{W}=-0.5, \omega=2$ (we have basically the same features for $k="+1 ", \epsilon=+1, \omega<$ $\left.0, \omega<2 \Lambda_{W}\right)$. The plots show that the curvature singularity $r_{S}$ is always inside the inner horizon $r_{-}$.

such a case the surface-like singularity may be hidden depending on the value of the parameters (figure 5 (right)). This leaves us with the following two viable cases.

(e) $2 \Lambda_{W}<\omega \leq \Lambda_{W}<0, \epsilon=+1$ : here, whenever $\beta>\beta_{*}$, there exists a cosmological horizon $r_{+}$as well as a black membrane horizon $r_{-}$(figure 5 (right), figure 7 (left)). The point-like singularity at $r=0$ is hidden by the black membrane horizon $r_{-}$with the observer region $r_{-}<r<r_{+}$. The curvature singularity at $r_{S}$ is always beyond the cosmological horizon $r_{+} \leq r_{S}$.

(f) $2 \Lambda_{W}<\Lambda_{W}<\omega<0, \epsilon=+1$ : in this case there exists a black membrane horizon $r_{+}$ when $\beta>\beta_{*}$ so that the point-like singularity at $r=0$ is also hidden, as in the case (e). However, in contrast to that case the surface-like singularity can be hidden only for $\beta \leq$ $\tilde{\beta}$, where $\tilde{\beta}$ is defined as the value of $\beta$ for which there is a merging of the cosmological horizon with the surface-like singularity, $r_{S}=r_{+} \equiv \tilde{r}_{+}$for $\beta=\tilde{\beta}$ (figure 5 (right), figure 7 (center)). For $\beta>\tilde{\beta}$, there is no cosmological horizon at $r_{+}$behind which the surface-like singularity would be hidden; this can be seen in the absence of the larger root for $f(r)=0$ (figure 5 (right), top curve). ${ }^{14}$ So, this is the case in which the surfacelike singularity can penetrate to our observer region unless $\beta$ is constrained as $\beta \leq \tilde{\beta}$. ${ }^{15}$

B. Case $\boldsymbol{\beta}<\mathbf{0}$ : the situation is quite different for the $\beta<0$ case, where viable black membrane solutions exist in the following exceptional case

(c') $\omega>0, \omega>2 \Lambda_{W}, \epsilon=-1$ : this is the case where $\beta$ can be negative so that we have a chance to satisfy $0>\beta>\beta_{*}$ with the inner and outer black hole horizon at $r_{ \pm}$ (figure 8 (left), figure 9 (left)).

\footnotetext{
${ }^{14}$ In this case again, the root formula for $r_{+}$in (3.16) does not apply due to the same reason as in the footnote 12 .

${ }^{15}$ This phenomena may be interpreted as the horizons being melted away or eaten by the surface-like singularity since the latter carries infinite temperature as can be seen in section IV.
} 
Here, it is important to note that even if the surface-like curvature singularity at $r_{S}$ is present, it sits always inside the black membrane horizon, i.e., $r_{+}>r_{S}$. Actually, as we increase $\beta$ from its minimum value $\beta_{*}<0$, the inner horizon $r_{-}$shrinks and meets the curvature singularity $r_{S}$ at $r=\tilde{r}_{-}, \beta=\tilde{\beta}$ (figure 7 (right)). On the other hand, for $\beta>\tilde{\beta}$, there is no inner horizon, but the curvature singularity at $r_{S}$ is not naked since the outer black membrane horizon is always outside the singularity, i.e., $r_{+}>r_{S}$. For $\beta<\beta_{*}$, the horizons are not formed so that the surface singularity is naked. This case flows to the exceptional solution of $M<0, \Lambda<0$ in GR.

\subsubsection{Spherical membrane solution $(k=+1)$}

Finally, the spherical membrane case $k=+1$ is known as the black hole solution. Its horizon structure can be understood as the intersections of the curves in figures 3 to 6 with an horizontal line at $f(r)=-1$. Similarly to the previous cases, we classify the solutions according to the sign of $\beta$.

A. Case $\boldsymbol{\beta}>\mathbf{0}$ : according to the same classification as before, we have the following viable cases.

(a) $\omega=0$ : here, the surface-like singularity does not exist. The point-like singularity at $r=0$ is hidden by an inner black hole horizon $r_{-}$as well as by an outer black hole horizon $r_{+}$, with the correct signature of the metric for $r>r_{+}$whenever $\beta>\beta_{*}$ in the AdS branch $(\epsilon=-1)$ (figure 3 (left)). Due to the absence of an inner horizon, in the dS branch $(\epsilon=+1)$, there are no viable solutions; the singularity at the origin is always naked as seen from the observer region $0<r<r_{+}$with a cosmological horizon $r_{+}$(figure 3 (right)).

(b) $\omega=2 \Lambda_{W}$ : this case corresponds to $-\Lambda_{W} \rightarrow \Lambda_{W}$ in the result of (a) and so all the properties can be understood just by flipping the sign of $\Lambda_{W}$.

(c) $\omega>0, \omega>2 \Lambda_{W}, \epsilon=-1$ : similar to the cases (a) and (b), the surface-like curvature singularity does not exist, and the point-like singularity at $r=0$ is hidden by an inner black hole horizon at $r_{-}$as well as by an outer black hole horizon at $r_{+}$whenever $\beta>\beta_{*}$, with the right signature of metric for the spatial coordinate $r>r_{+}$(figure 4 (left)). This case flows to the case $M>0, \Lambda<0$ of GR.

Notice that the case $\omega<0, \omega<2 \Lambda_{W}, \epsilon=+1$ is not viable for $k=+1$, since there is only a cosmological horizon $r_{+}$and so the singularity at $r=0$ is never hidden in the observer region $0 \leq r<r_{+}$(figure 4 (right)). On the other hand, for the cases in which there is a surface-like curvature singularity at $r_{S}$, we can either have no horizon, or have a cosmological horizon at $r_{+}$(case $2 \Lambda_{W}<\omega<0, \epsilon=+1$ ), but then the point-like singularity at $r=0$ is naked as seen from our observer region (figure 5 (right)); or have a black hole horizon (case $2 \Lambda_{W}>\omega>0, \epsilon=-1$ ), and in such case the surface-like singularity is naked (figure 5 (left)). This leaves us with no viable cases. 

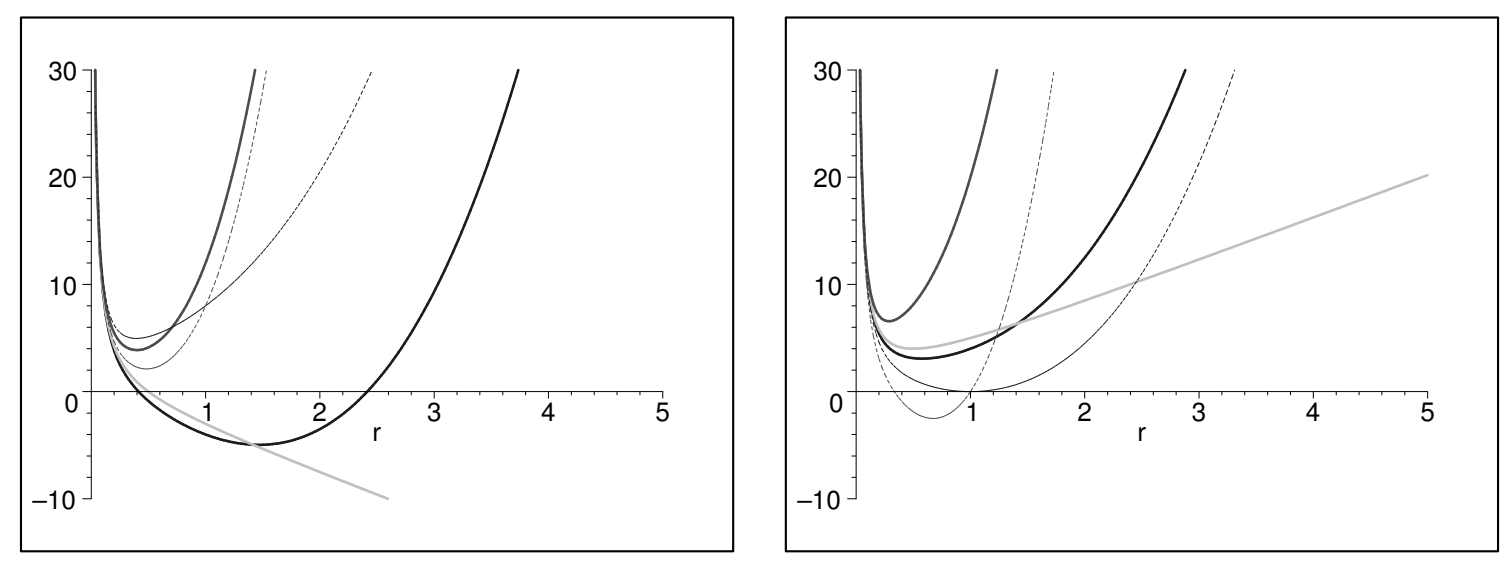

Figure 8. Plots of $\beta$ vs. $r_{+}$. The thick curves denote the hyperbolic membrane $(k=-1)$ for $\Lambda_{W}=-1,0,3$ (bottom to top, from left) and thin dotted curves denote the spherical membrane $(k=+1)$ for $\Lambda_{W}=-1,3$ (top to bottom, from left). Left: case $\omega>0$. For the $k=-1$ case, $\beta$ can be negative so that we have a chance to satisfy the condition $\beta_{*}<\beta<0$ for the existence of horizons $r_{ \pm}$with $\beta<0$. However, this is not possible for the $k=+1$ case. The plots are for $\omega=2$. Right: case $\omega<0$. Here, the situation is the opposite and for the $k=+1$ case, $\beta$ can be negative so that we have a chance to satisfy the condition $\beta_{*}<\beta<0$ for the existence of horizons $r_{ \pm}$with $\beta<0$. However, this is not possible for the $k=-1$ case. The plots are for $\omega=-2$.
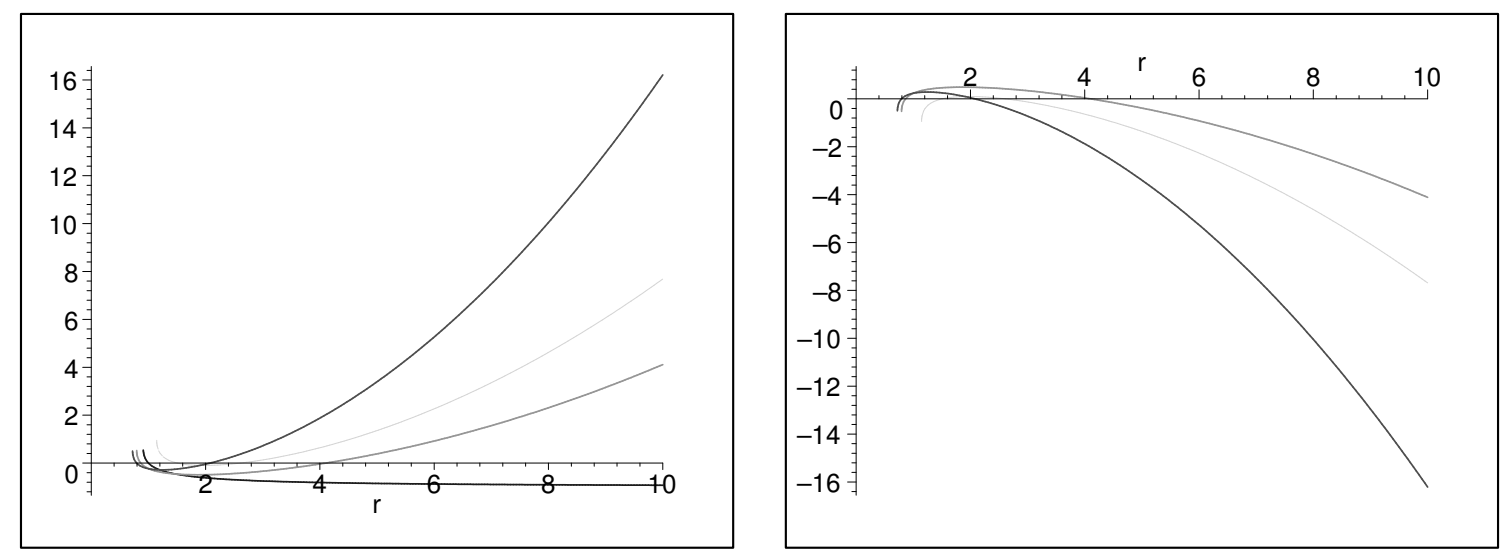

Figure 9. Plots of $f(r)$ for $\beta<0, \omega\left(2 \Lambda_{W}-\omega\right)<0$. Left: case $k=-1, \epsilon=-1, \omega>0, \omega>$ $2 \Lambda_{W}$. The curves denote black hyperbolic branes with the inner and outer horizons for $\Lambda_{W}=$ $-1,-0.5,0,0.5, \omega=2(\beta=-3)$ from left to right, respectively. Right: case $k=+1, \epsilon=+1, \omega<$ $0, \omega<2 \Lambda_{W}$. The curves denote black membranes with the inner and outer horizons for $\Lambda_{W}=$ $1,0.5,-0.5, \omega=-2(\beta=-3)$ from left to right, respectively.

B. Case $\boldsymbol{\beta}<\mathbf{0}$ : for $\beta<0$ in the AdS branch $(\epsilon=-1)$, we have the same situation as in the $k=0$ case, having no horizon and no viable solutions without naked singularity (figure 6 (left)). In the dS branch $(\epsilon=+1)$, there is a curvature singularity $r_{S}$ at the boundary of the real metric with $\omega<0, \omega<2 \Lambda_{W}$ and otherwise, there is no real-valued metric for the whole region as in the $k=0$ case. This leaves us with the only interesting one as follows.

(d') $\omega<0, \omega<2 \Lambda_{W}, \epsilon=+1$ : in this case, an inner black hole horizon at $r_{-}$and an outer cosmological horizon at $r_{+}$exist, as long as $\beta>\beta_{*}$ (figure 8 , figure 9 (right)), and the 
observer region is $r_{-}<r<r_{+}$. This case flows to the case $M>0, \Lambda>0$ of GR. As we increase $\beta$ from its minimum value $\beta_{*}<0$, the black hole horizon $r_{-}$shrinks and meets the curvature singularity $r_{S}$ at $r=\tilde{r}_{-}, \beta=\tilde{\beta}$ (figure 7 (right)). On the other hand, for $\beta>\tilde{\beta}$, there is no black hole horizon so that the curvature singularity at $r_{S}$ becomes naked. This situation is analogous to that of $k=-1$ in figure 7 (center), but now the surface-like singularity can penetrate to our observer region from inside the black hole horizon unless $\beta$ is constrained as $\beta \leq \tilde{\beta}$. This achieves a close analogy with the singularity at $r=0$ in GR case, which can be naked unless $M$ is constrained as $M>0$ for $\Lambda>0$.

To conclude this section, we have classified all the viable $(\lambda=1)$ static black membrane solutions without naked curvature singularities as seen from the observer region, where the metric has the right signature for Hořava gravity. The solutions are classified by $\omega, \Lambda_{W}$, and $\beta$, and we have found several interesting black membrane solutions which do not exist in GR. In particular, we have found that there are black plane $(k=0)$ and hyperbolic $(k=-1)$ branes even in the dS branch (case (d), (e) for the former and case (d), (e), (f) for the latter), where there is a cosmological horizon, as well as in the AdS branch. This implies that, in these particular cases, some additional "attraction" is generated due to the higher-derivative effects of Hořava gravity so that the membranes can be formed by overcoming the global repulsion in the dS branch.

\section{Thermodynamics}

For the AdS branch, the solution (3.10) has two horizons generically and the Hawking temperature for the outer horizon $r_{+}$is given by ${ }^{16}$

$$
T_{H}=\frac{3 \Lambda_{W}^{2} r_{+}^{4}+2 k\left(\omega-\Lambda_{W}\right) r_{+}^{2}-k^{2}}{8 \pi r_{+}\left(k+\left(\omega-\Lambda_{W}\right) r_{+}^{2}\right)} .
$$

Note that this temperature diverges when the horizon radius $r_{+}$coincides with the point-like singularity at $r=0$ and, interestingly, also when it coincides with the surfacelike singularity at $r_{S}$, where the denominator vanishes.

Figure 10 (left) shows that the black hole temperature interpolates between the asymptotically AdS cases (above three curves) and flat (bottom curve) case. There exists an extremal black hole limit of vanishing temperature where the inner horizon $r_{-}$meets with the outer horizon $r_{+}$at $r=r_{*}$ and the integration constant $\beta$ gets its minimum $\beta_{*}$. For smaller black holes of $r_{+}<r_{*}$, the black hole temperature becomes negative, implying a thermodynamics instability. This may provide the minimum size for a thermodynamically

\footnotetext{
${ }^{16}$ Due to the lack of Lorentz invariance in UV, the very meaning of the horizons and Hawking temperature would be changed from the conventional ones. The light cones would differ for different wavelengths and so different particles with different dispersion relations would see different Hawking temperature and entropies, and the Hawking spectrum would not be thermal. But from the recovered Lorentz invariance in the IR (with $\lambda=1$ ), the usual meaning of the horizons and $T_{H}$ as the Hawking temperature would be "emerged" for long wavelengths. The calculation and meaning of the temperature should be understood in this context.
} 

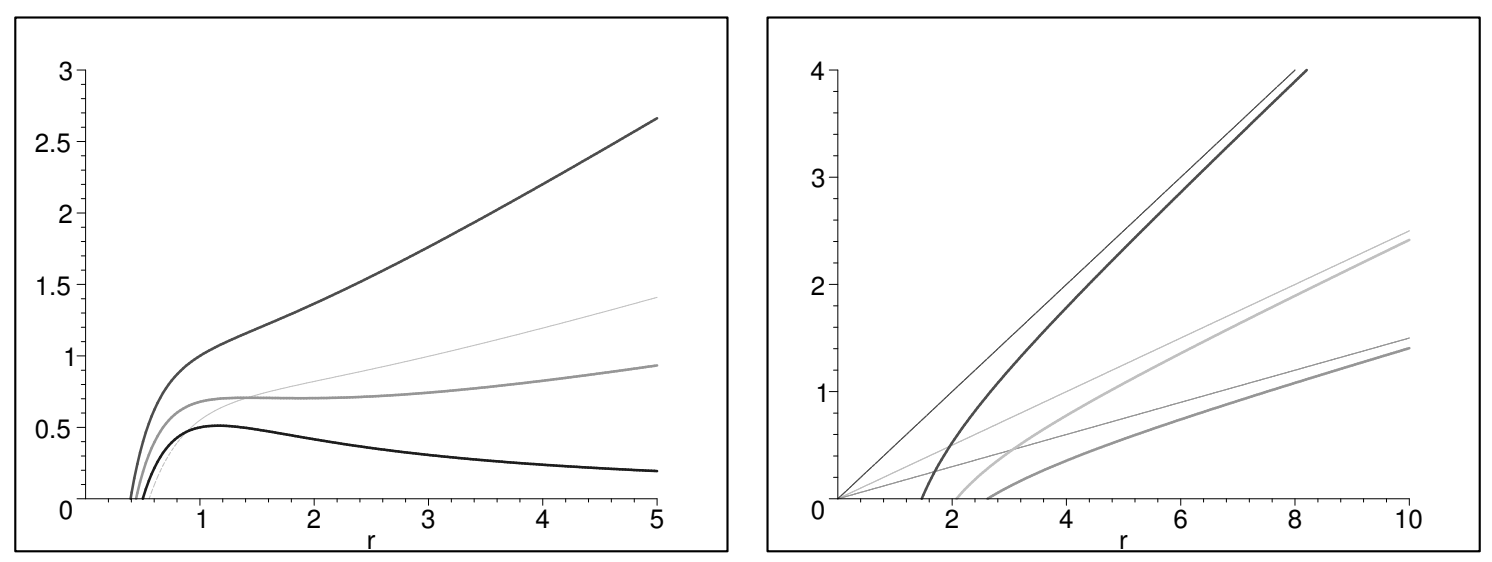

Figure 10. Plots of $4 \pi T$ vs. the outer horizon radius $r_{+}$for the AdS branch with $\omega>0, \omega>2 \Lambda_{W}, \epsilon=-1$. Left: black hole case $(k=+1)$, in particular for $\Lambda_{W}=-1,-0.5,0$ (top to bottom, thick curves), 0.5 (thin curve), $\omega=2$. Right: hyperbolic membrane case $(k=-1)$ (thick curves), and flat membrane case $(k=0)$ (thin curves), in particular for $\Lambda_{W}=-1$ (top), -0.5 (bottom), 0.5 (middle), $\omega=2$.

stable black hole. The flat $(k=0)$ and hyperbolic $(k=-1)$ membranes have the same properties (figure 10 (right)), even though we have zero minimum radius for $k=0$.

For the dS branch, the solution (3.10) can have the inner black hole/membrane horizon at $r_{-}$and the cosmological horizon at $r_{+}$, and the temperature for the black hole/membrane is given by (4.1) also but now with $r_{-}$in place of $r_{+}$. There is an extremal limit of vanishing temperature at $r_{+}=r_{-}=r_{*}$ in which the black hole/membrane horizon coincides with the cosmological horizon, i.e., the Nariai limit (figure 11). We see that the temperature becomes infinity at the vanishing limit of membrane radius where the black membrane horizon at $r_{-}$meets with the point-like singularity at $r=0$, as in the case of Schwarzschild or Schwarzschild-de Sitter black hole in GR (figure 11 (center)). It is interesting to note that there is another (positive) infinite temperature point at $r=\tilde{r}_{ \pm}$where the black membrane horizon at $r_{-}$or the cosmological horizon at $r_{+}$coincides with the surface-like singularity at $r_{S}$, which provides lower/upper bounds for the black hole/cosmological horizons radius (figure 11 (left) and (right)). So, the occurrence of the infinite temperature would be a reflection of the coincidence of a curvature singularity with the black hole/membrane or cosmological horizons. ${ }^{17}$

Finally, we note that one can also consider the first law of black membrane thermodynamics as in the usual form, for the black membrane horizon $r_{+}$

$$
d \mathcal{M}=T_{H} d \mathcal{S},
$$

with the black membrane's mass and entropy

$$
\mathcal{M}=\frac{\kappa^{2} \mu^{2} \Omega_{k} \beta}{16}
$$

\footnotetext{
${ }^{17}$ This seems to be quite generic behaviors when the Killing and apparent horizons coincide. But, this does not be seems to be true otherwise. See for example [63].
} 

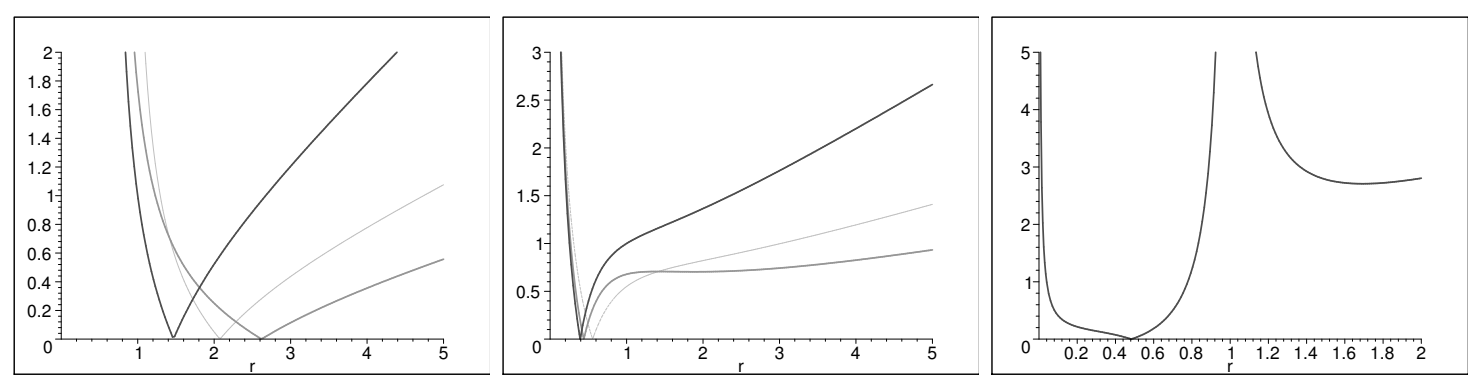

Figure 11. Plots of $4 \pi T$ vs. black hole or membrane's horizon radius $r_{-}$(left part) and cosmological horizon radius $r_{+}$(right part) for the dS branch $(\epsilon=+1)$. Left: black hole case $(k=+1)$ with $\omega<0, \omega<2 \Lambda_{W}$, in particular for $\Lambda_{W}=1,0.5$ (top to bottom, thick curves), $\Lambda_{W}=-0.5$ (thin curve), $\omega=-2$. The black hole temperature becomes infinity at $\tilde{r}_{-}$where the black hole's horizon coincides with the surface-like singularity. Center: hyperbolic membrane case $(k=-1)$ with $\omega<0, \omega<2 \Lambda_{W}$, in particular for $\Lambda_{W}=1,0.5$ (top to bottom, thick curves), $\Lambda_{W}=-0.5$ (thin curve), $\omega=-2$. Right: hyperbolic membrane case with $\Lambda_{W}<\omega<0$, in particular for $\Lambda_{W}=-3, \omega=-2$. The $\overline{c o s m o l o g i c a l}$ horizon temperature becomes infinity at $\tilde{r}_{+}=1$, where the cosmological horizon coincides with the surface-like singularity.

$$
\mathcal{S}=\frac{\pi \kappa^{2} \mu^{2} \Omega_{k}}{4}\left(\left(\omega-\Lambda_{W}\right) r_{+}^{2}+2 k \ln r_{+}\right)+\mathcal{S}_{0}
$$

respectively, up to an arbitrary constant $\mathcal{S}_{0}[25,43]$. However, as far as we know, the very meaning of the entropy in Hořava gravity is not quite clear and not well established yet [8].

\section{Connection to time-dependent cosmological solutions}

So far, we have studied the viable solutions, without naked singularities, of black holes and black membranes with dS or AdS asymptotics, for $\lambda=1$, which matches with GR in the IR. In GR, there is a close connection between a static metric and a time-dependent cosmological solution via coordinate transformations which mix space and time. For example, the $d S_{4}$ metric in static coordinates can be mapped into a flat FLRW metric in planar coordinates [64]. Since Einstein equations are invariant under such change of coordinates, the static solutions are mapped into cosmological solutions. However, in Hořava gravity, this correspondence does not hold anymore, due to lack of full diffeomorphism invariance, and we cannot get a direct connection between those two spacetimes. In this section, we study whether some information, in particular the conditions for the viable solutions without naked singularities, can be mapped from static black holes or membranes to cosmology solutions, even in the absence of a direct connection. To this end, we consider a homogeneous and isotropic cosmological ansatz for the action (3.2) with the standard FLRW form

$$
d s^{2}=-c^{2} d t^{2}+a^{2}(t)\left(\frac{d r^{2}}{1-\mathbf{k} r^{2}}+r^{2}\left(d \theta^{2}+\sin ^{2} \theta d \phi^{2}\right)\right)
$$


where the three-dimensional spatial curvature $\mathbf{k}=+1,0,-1$ correspond to a closed, flat, and open universe, respectively. The curvature invariants of the metric (5.1) are given by

$$
R^{(3)}=\frac{6 \mathbf{k}}{a^{2}}, \quad K_{i}^{i}=\frac{3 \dot{a}}{a}
$$

and we see that there is only an initial curvature singularity at $a(t)=0$.

Assuming the matter contribution to be of the form of a perfect fluid with the energy density $\rho$ and pressure $p$, we find that

$$
\begin{aligned}
\left(\frac{\dot{a}}{a}\right)^{2} & =\frac{\kappa^{2}}{6(3 \lambda-1)}\left[\rho-\frac{3 \kappa^{2} \mu^{2}}{8(3 \lambda-1)}\left(\frac{\mathbf{k}^{2}}{a^{4}}+\frac{2 \mathbf{k}\left(\omega-\Lambda_{W}\right)}{a^{2}}+\Lambda_{W}^{2}\right)\right], \\
\frac{\ddot{a}}{a} & =\frac{\kappa^{2}}{6(3 \lambda-1)}\left[-\frac{1}{2}(\rho+3 p)+\frac{3 \kappa^{2} \mu^{2}}{8(3 \lambda-1)}\left(\frac{\mathbf{k}^{2}}{a^{4}}-\Lambda_{W}^{2}\right)\right] .
\end{aligned}
$$

Note that the $1 / a^{4}$ term, which is the contribution from the higher-derivative terms in the action (3.2), exists only for $\mathbf{k} \neq 0$ and becomes dominant for small $a(t)$, implying that the cosmological solutions of GR are recovered at larger scales. As usual, the second equation has a first integral, whose value is completely fixed by the first, which turns out to be the only independent equation of the system. Here, we have not restricted to $\lambda=1$ like the previous sections since the following analysis is more generally valid for arbitrary values of $\lambda>1 / 3$, which would be quite useful in cosmology [4-6].

In order to study the solutions for the scale factor $a(t)$, it is useful to consider the effective potential

$$
V_{\mathrm{eff}}=\frac{\kappa^{2}}{32(3 \lambda-1)^{2}}\left[-2(3 \lambda-1) \rho a^{2}+\kappa^{2} \mu^{2}\left(\frac{\mathbf{k}^{2}}{a^{2}}+2 \mathbf{k}\left(\omega-\Lambda_{W}\right)+\Lambda_{W}^{2} a^{2}\right)\right],
$$

on the effective mechanical equation $\dot{a}^{2} / 2+V_{\text {eff }}=0$ for a particle of unit mass and zero energy. In this picture, a non-singular cosmology corresponds to a situation in which there are bouncing points that prevent the particle to reach the origin $a=0$. The bouncing points are located at the values of the scale factor at which $\dot{a}^{2} / 2=-V_{\text {eff }}=0$.

For the case of a flat $(\mathbf{k}=0)$ universe solution, there is no contribution to the effective potential arising from the higher-derivative terms in Hořava gravity, so that we have basically the same situation as in GR where the initial singularity exists always ${ }^{18}$ unless we introduce some exotic matter that violates energy conditions, i.e., $\rho<0$.

However, for the non-flat $(\mathbf{k} \neq 0)$ cases, non-singular vacuum cosmology solutions can exist $[5]^{19}$ if the following conditions,

$$
\omega\left(\omega-2 \Lambda_{W}\right) \geq 0, \quad \mathbf{k}\left(\omega-\Lambda_{W}\right)<0
$$

are satisfied. In such a case, the bouncing points for $V_{\text {eff }}=0$ exist, at the values of the scale factor given by

$$
\left(a^{ \pm}\right)^{2}=\frac{-\mathbf{k}\left(\omega-\Lambda_{W}\right) \pm \sqrt{\mathbf{k}^{2} \omega\left(\omega-2 \Lambda_{W}\right)}}{\Lambda_{W}^{2}} .
$$

\footnotetext{
${ }^{18}$ For the de Sitter-type universe solution, with a exponentially growing or decaying scale factor $a(t)$, the singularity, $a(t)=0$, is pushed to the infinite past $t \rightarrow-\infty$ or the infinite future $t \rightarrow+\infty$, respectively but the Big Bang or Big Crunch singularity problem remains still.

${ }^{19}$ For the non-singular cosmology solutions in the presence of matter, see [65-67].
} 
More explicitly, for the AdS/flat branch with $\mu^{2}>0, \omega>0$, in particular, for $\Lambda_{W} \neq 0$, the general solution for arbitrary $\mathbf{k}$ is given by

$$
a_{\mathrm{AdS}}^{2}(t)=\frac{-\mathbf{k}\left(\omega-\Lambda_{W}\right)}{\Lambda_{W}^{2}}\left[1+\sqrt{\frac{\omega\left(\omega-2 \Lambda_{W}\right)}{\left(\omega-\Lambda_{W}\right)^{2}}} \cos \left(\frac{\kappa^{2} \mu \Lambda_{W}}{2(3 \lambda-1)}(t-\gamma)\right)\right] .
$$

For $\omega>2 \Lambda_{W}, \mathbf{k}\left(\omega-\Lambda_{W}\right)<0$, it admits a non-singular cyclic cosmology solution, which is oscillating between the inner and outer bouncing scale factors, $a^{-}$and $a^{+}$, respectively, in (5.7), with an integration constant $\gamma$ depending on the initial conditions. Notice that in this case, the second condition in (5.6) can be satisfied only for $\mathbf{k}=-1$. In the case $\Lambda_{W}=0$, on the other hand, the general solution is given by

$$
a_{\text {flat }}^{2}(t)=-\frac{\kappa^{4} \mu^{2} \mathbf{k} \omega}{8(3 \lambda-1)^{2}}(t-\gamma)^{2}-\frac{\mathbf{k}}{2 \omega}
$$

and, for $\mathbf{k}=-1$, this admits a non-singular cosmology solution with only one bouncing at the scale factor $a^{-}$(figure 12 (left)). Moreover, in the case $\omega=0$, or $2 \Lambda_{W}$, the two bouncing points meet and there exists only a static cosmology solution of $a^{2}=\mathbf{k} / \Lambda_{W}$ or $-\mathbf{k} / \Lambda_{W}$ when $\mathbf{k}=-1$ or +1 , respectively, and $\Lambda_{W}<0$ [3].

On the other hand, for the dS branch with $\mu^{2}<0, \omega<0$, the general solution for arbitrary $\mathbf{k}$ is given by

$$
a_{\mathrm{dS}}^{2}(t)=\frac{2|3 \lambda-1|}{\kappa^{2}|\mu|\left|\Lambda_{W}\right|} \mathrm{e}^{ \pm \frac{\kappa^{2}|\mu|\left|\Lambda_{W}\right|}{2|3 \lambda-1|}(t-\gamma)}+\frac{\mathbf{k}^{2} \kappa^{2}|\mu| \omega\left(\omega-2 \Lambda_{W}\right)}{8|3 \lambda-1|\left|\Lambda_{W}\right|^{3}} \mathrm{e}^{\mp \frac{\kappa^{2}|\mu|\left|\Lambda_{W}\right|}{2|3 \lambda-1|}(t-\gamma)}-\frac{\mathbf{k}\left(\omega-\Lambda_{W}\right)}{\Lambda_{W}^{2}} .
$$

For $\omega<2 \Lambda_{W}, \mathbf{k}\left(\omega-\Lambda_{W}\right)<0$, the solution admits a non-singular universe which has one bouncing at $a^{+}$when $a(t)$ shrinks toward $a^{+}$from larger values and the universe evolves to $d S_{4}$ vacuum. Notice that now the second condition in (5.6) can be satisfied only for $\mathbf{k}=+1$. Otherwise, the initial singularity exists always. For example, for $\omega>2 \Lambda_{W}$ so that the first condition in (5.6) is not satisfied, there is a singular solution with a bounce at $a^{-}$when $a(t)$ expands toward $a^{-}$from smaller values and then shrink towards the initial singularity ${ }^{20}$ (figure 12 (right)). Moreover, in the case $\omega=0$ or $2 \Lambda_{W}$, the two bouncing points meet at $a^{2}=\mathbf{k} / \Lambda_{W}$ or $-\mathbf{k} / \Lambda_{W}$ but it admits the universe which evolves monotonically from that minimum scale factor to $d S_{4}$ vacuum asymptotically or vice versa [3, 5].

Before concluding this section, we note that, as mentioned above, the general (vacuum) cosmological solutions for arbitrary $\mathbf{k}$ in GR can be consistently obtained from the GR limit $(17)-(19)$ as follows:

$$
a_{\text {flat }}(t)=\sqrt{-\mathbf{k} c^{2}}(t-\gamma) \quad \text { for } \Lambda=0,
$$

\footnotetext{
${ }^{20}$ For $\mathbf{k} \neq 0$, the solution $(5.10)$ reduces to $a_{\mathrm{dS}}^{2}(t)=\left(-\mathbf{k}\left(\omega-\Lambda_{W}\right) / \Lambda_{W}^{2}\right)\{1+$ $\left.\sqrt{\omega\left(\omega-2 \Lambda_{W}\right) /\left(\omega-\Lambda_{W}\right)^{2}} \cosh \left[\left(\kappa^{2}|\mu|\left|\Lambda_{W}\right| / 2(3 \lambda-1)\right)(t-\gamma)\right]\right\} \quad$ or $a_{\mathrm{dS}}^{2}(t)=\left(-\mathbf{k}\left(\omega-\Lambda_{W}\right) / \Lambda_{W}^{2}\right)\{1 \pm$ $\left.\sqrt{\omega\left(-\omega+2 \Lambda_{W}\right) /\left(\omega-\Lambda_{W}\right)^{2}} \sinh \left[\left(\kappa^{2}|\mu|\left|\Lambda_{W}\right| / 2(3 \lambda-1)\right)(t-\gamma)\right]\right\}$ by shifting the integration constant $\gamma$, $\exp \left[\mp\left(\kappa^{2}|\mu|\left|\Lambda_{W}\right| / 2|3 \lambda-1|\right) \gamma\right] \rightarrow\left\{\kappa^{2}|\mu||\mathbf{k}| \sqrt{\omega\left(\omega-2 \Lambda_{W}\right)} / 4|3 \lambda-1|\left|\Lambda_{W}\right|\right\} \quad \exp \left[\mp\left(\kappa^{2}|\mu|\left|\Lambda_{W}\right| / 2|3 \lambda-1|\right) \gamma\right]$ or $\left\{\kappa^{2}|\mu||\mathbf{k}| \sqrt{\omega\left(-\omega+2 \Lambda_{W}\right)} / 4|3 \lambda-1|\left|\Lambda_{W}\right|\right\} \exp \left[\mp\left(\kappa^{2}|\mu|\left|\Lambda_{W}\right| / 2|3 \lambda-1|\right) \gamma\right]$ for $\omega<2 \Lambda_{W}$ or $\omega>2 \Lambda_{W}$, respectively. Here, the former non-singular case corresponds to the analytic continuation from the AdS branch solution (5.8), but a similar continuation is absent for the latter singular case. For $\mathbf{k}=0$, on the other hand, (5.10) reduces to the exponentially growing or decaying solution also.
} 

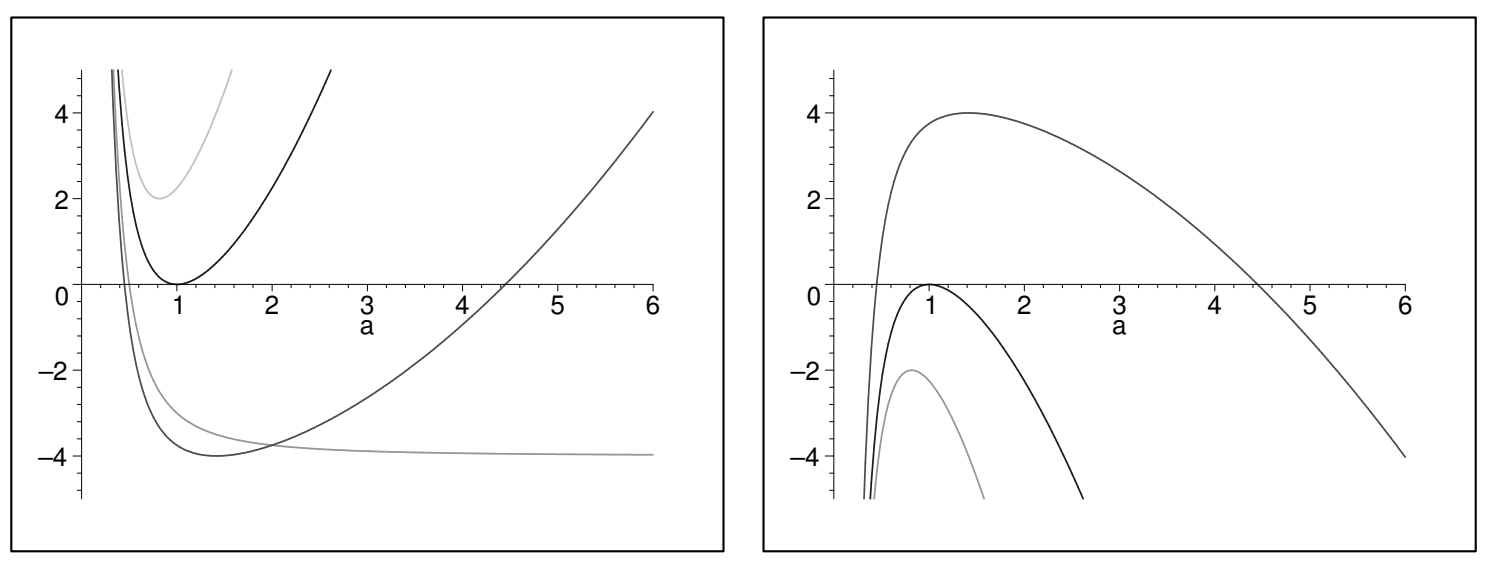

Figure 12. Plots of the vacuum effective potential $V_{\text {eff }}$ vs. scale factor $a(t)$. Left: case $\omega>0, \mathbf{k}=$ -1 , in particular $\Lambda_{W}=-0.5,0,1,1.5, \omega=2$ (bottom to top) with $\kappa^{4} \mu^{2} / 32(3 \lambda-1)^{2} \equiv 1$. The dynamical cosmology solutions exist only for $\omega\left(\omega-2 \Lambda_{W}\right)>0,\left(\omega-\Lambda_{W}\right)>0$ and there are no initial singularities at $a=0$. There are one bounce at $a^{-}$and another at $a^{+}$for $\Lambda_{W} \neq 0$ so that the universe becomes cyclic. But there is only one bounce at $a^{-}$for $\Lambda_{W}=0$ and so no cyclic universe exists. Right: case $\omega<0, \mathbf{k}=+1$, in particular $\Lambda_{W}=0.5,-1,-1.5, \omega=-2$ (top to bottom) with $\left.\kappa^{4} \mu^{2} / 3 \overline{2(3 \lambda}-1\right)^{2} \equiv-1$. The non-singular cosmology solutions, which have a bounce at $a^{+}$, exist only for $\omega\left(\omega-2 \Lambda_{W}\right)>0,\left(\omega-\Lambda_{W}\right)<0$ (top curve).

$$
\begin{array}{rlr}
a_{\mathrm{AdS}}(t) & =\sqrt{-\frac{3 \mathbf{k} c^{2}}{|\Lambda|}} \cos \left(\sqrt{\frac{|\Lambda|}{3}}(t-\gamma)\right) & \text { for } \Lambda<0 \\
a_{\mathrm{dS}}(t) & =\frac{1}{\sqrt{12 \Lambda}}\left(\sqrt{6 \sqrt{3 \Lambda}} \mathrm{e}^{ \pm \sqrt{\Lambda / 3}(t-\gamma)}+\frac{9 \mathbf{k} c^{2}}{\sqrt{6 \sqrt{3 \Lambda}}} \mathrm{e}^{\mp \sqrt{\Lambda / 3}(t-\gamma)}\right) & \text { for } \Lambda>0 .
\end{array}
$$

In the last case, the solution reduces to the usual form of $a_{\mathrm{dS}}(t)=\sqrt{3 c^{2} / \Lambda} \cosh (\sqrt{\Lambda / 3}(t-$ $\gamma)$ ) or $a_{\mathrm{dS}}(t)= \pm \sqrt{3 c^{2} / \Lambda} \sinh (\sqrt{\Lambda / 3}(t-\gamma))$ by the integration constant shift of $\mathrm{e}^{\mp \sqrt{\Lambda / 3}} \gamma \rightarrow$ $(3 c / \sqrt{6 \sqrt{3 \Lambda}}) \mathrm{e}^{\mp \sqrt{\Lambda / 3}} \gamma$ for $\mathbf{k}=+1$ or $\mathbf{k}=-1$, respectively. The recovery of cosmological solutions in GR, similarly to the black hole solutions in the previous sections, is not possible in the absence of $\omega$, i.e., in the original Hořava gravity with the detailed balance condition [3].

To conclude this section, we have classified the non-singular vacuum FLRW cosmology solutions in Hořava gravity for the non-flat $(\mathbf{k} \neq 0)$ universe, by the condition (5.6). Note that the conditions agree with the condition $\omega\left(\omega-2 \Lambda_{W}\right) \geq 0$ for the non-singular static black hole/membrane geometry in section II. And also from (5.6), we have found some intimate relation between $\mathbf{k}$ and $\omega$, i.e., $\mathbf{k}=-1$ for $\omega>\Lambda_{W}, \mathbf{k}=+1$ for $\omega<\Lambda_{W}$ for the non-singular cosmology solutions.

\section{Concluding remarks}

We have studied the singularity and horizon structures of the static black hole and membrane solutions in IR-modified Hořava gravity, and classified all the viable solutions without naked singularities. We have found a physical picture that is quite different from the conventional one. In particular, we have found that, in addition to the usual point-like singularity at the origin, there is a surface-like singularity that becomes the cutting edge of space-time, where the real-valued metric ends and unconventional complex-valued metric 
starts. The degrees of divergence of curvature on such singularities is milder than that of GR. Moreover, the Hawking temperature of the horizon is finite, unless any of the singularities coincide with the the outermost horizon. We also found that there are viable black plane $(k=0)$ and hyperbolic $(k=-1)$ brane solutions even in the dS branch, where there is a cosmological horizon, as purely higher-derivative effects of Hořava gravity. We have also found some consistency with the conditions for non-singular time-dependent cosmological solutions. Several further remarks are in order.

First, according to Hořava gravity's idea for curing the renormalizability problem without ghosts, we need the higher-spatial derivative terms while keeping quadratic in timederivatives. However, wherever the lapse function becomes negative, as it happens in the region inside the outer black hole horizon or beyond the cosmological horizon, we have highertime derivatives while keeping quadratic in space-derivatives instead. But it is known that the higher-time derivatives would produce the so called Ostrogradsky instability. The detailed analysis would be beyond the scope of this paper, but we suspect that this may be not harmful at the classical level inside the black hole/membrane horizons due to the finite range in the "time"-coordinate $r$, that would prevent that a runaway behavior lasts enough time to develop the infinite growth in any perturbations. However, the problem persists beyond a cosmological horizon, if the real space-time does not end at some finite time $r_{S}$.

Second, the black plane solutions that we have studied can be also considered as the black "string" solutions if we make a compactification along one direction on the plane. ${ }^{21}$ It is well known that there is Gregory-Laflamme instability in higher-dimensional black strings for Einstein gravity. So, it would be interesting to investigate the similar instability in our four dimensional black plane solutions.

Third, the notion of horizon in Hořava gravity might be subtle, because it depends on the dispersion relation of probing particles/fields. In particular, if we consider the gravitational perturbations inside the horizon, they can leak out from the horizon due to its non-relativistic dispersions for high momentum, so that one can probe the singularities inside the horizon. On the other hand, since the degree of singularity is milder than that of GR, it would be interesting to investigate whether it is possible to get some non-singular information via dispersive gravitons.

Fourth, we have found some interesting agreements in the conditions for non-singular static black hole or membrane metric with non-singular cosmology solutions. We do not know whether this is just a coincidence or there is some more fundamental reason which is not clear in our formulation. Moreover, there is another type of correspondence which connects the domain-wall and cosmology solutions via complex coordinate transformations which mix space and time in GR [70] but does not hold anymore in Hořava gravity, due to lack of the symmetry between space and time. If there is some more fundamental reason for the obtained agreements, we may conjecture that a similar agreements may be found in this case also. It would be interesting to see whether there exits a similar correspondence between the non-projectable and projectable theories, which are known to be quite distinct in the original Hořava gravity setup that are adopted in this paper.

\footnotetext{
${ }^{21}$ A certain class of the black string solutions, where the Cotton tensor vanishes, in the original Hor̆ava gravity with the detailed balance condition are already known [68, 69], but the general class of the black string solutions with/without the detailed balance condition are not known yet.
} 
Finally, we have shown the importance of the "GR limit", which achieves a peculiar form of flows of coupling constants, in order to recover the results of GR in the asymptotic region. We do not have any fundamental understanding about such flow yet. In particular, it would be interesting to understand the relation of this flow and that of Renormalization Group.

\section{Acknowledgments}

CRA and MIP are grateful to the organizers of the 13th Italian-Korean meeting on Relativistic Astrophysics at Ewha Womans University, for providing a proper scientific environment which led to the original idea of this collaboration. NEG thanks Diana Lopez Nacir and Gastón Giribet, and MIP would like to thank Shinsuke Kawai and Gungwon Kang for helpful comments. The authors are grateful to SISSA and ICTP for providing an extremely conformable work environment in which this work was started.

CRA was supported by the International Ceneter for Relativistic Astrophysics (ICRANet). NEG is supported by PIP CONICET grants $\mathrm{N}^{\circ} 0396$ and 0595 and UNLP project X648. MIP was supported by Basic Science Research Program through the National Research Foundation of Korea (NRF) funded by the Ministry of Education (2-2013-4569001-1).

Open Access. This article is distributed under the terms of the Creative Commons Attribution License (CC-BY 4.0), which permits any use, distribution and reproduction in any medium, provided the original author(s) and source are credited.

\section{References}

[1] P. Hořava, Membranes at quantum criticality, JHEP 03 (2009) 020 [arXiv:0812.4287] [INSPIRE].

[2] P. Hořava, Quantum gravity at a Lifshitz point, Phys. Rev. D 79 (2009) 084008 [arXiv:0901.3775] [INSPIRE].

[3] H. Lü, J. Mei and C.N. Pope, Solutions to Hořava gravity, Phys. Rev. Lett. 103 (2009) 091301 [arXiv: 0904.1595] [INSPIRE].

[4] A. Kehagias and K. Sfetsos, The black hole and FRW geometries of non-relativistic gravity, Phys. Lett. B 678 (2009) 123 [arXiv:0905.0477] [INSPIRE].

[5] M.-I. Park, The black hole and cosmological solutions in IR modified Horaava gravity, JHEP 09 (2009) 123 [arXiv: 0905.4480] [inSPIRE].

[6] M.-I. Park, A test of Horava gravity: the dark energy, JCAP 01 (2010) 001 [arXiv: 0906.4275] [INSPIRE].

[7] C.R. Arguelles and N.E. Grandi, Domain wall solutions to Hor̆ava gravity, arXiv:1008.1915 [INSPIRE].

[8] R.-G. Cai, L.-M. Cao and N. Ohta, Topological black holes in Hořava-Lifshitz gravity, Phys. Rev. D 80 (2009) 024003 [arXiv: 0904.3670] [INSPIRE].

[9] A. Ghodsi, Toroidal solutions in Hořava gravity, Int. J. Mod. Phys. A 26 (2011) 925 [arXiv: 0905.0836] [INSPIRE]. 
[10] R.-G. Cai and A. Wang, Singularities in Hor̆ava-Lifshitz theory, Phys. Lett. B 686 (2010) 166 [arXiv: 1001.0155] [INSPIRE].

[11] S. Mukohyama, Scale-invariant cosmological perturbations from Hořava-Lifshitz gravity without inflation, JCAP 06 (2009) 001 [arXiv:0904.2190] [INSPIRE].

[12] G. Calcagni, Cosmology of the Lifshitz universe, JHEP 09 (2009) 112 [arXiv:0904.0829] [INSPIRE].

[13] T. Takahashi and J. Soda, Chiral primordial gravitational waves from a Lifshitz point, Phys. Rev. Lett. 102 (2009) 231301 [arXiv:0904.0554] [INSPIRE].

[14] E. Kiritsis and G. Kofinas, Hořava-Lifshitz cosmology, Nucl. Phys. B 821 (2009) 467 [arXiv: 0904.1334] [INSPIRE].

[15] J. Kluson, Branes at quantum criticality, JHEP 07 (2009) 079 [arXiv:0904.1343] [INSPIRE].

[16] R. Brandenberger, Matter bounce in Hor̆ava-Lifshitz cosmology, Phys. Rev. D 80 (2009) 043516 [arXiv: 0904.2835] [INSPIRE].

[17] H. Nastase, On IR solutions in Hoŕava gravity theories, arXiv:0904.3604 [INSPIRE].

[18] R.-G. Cai, Y. Liu and Y.-W. Sun, On the $z=4$ Hořava-Lifshitz gravity, JHEP 06 (2009) 010 [arXiv: 0904 .4104] [INSPIRE].

[19] Y.-S. Piao, Primordial perturbation in Hořava-Lifshitz cosmology, Phys. Lett. B 681 (2009) 1 [arXiv: 0904 . 4117] [INSPIRE].

[20] X. Gao, Cosmological perturbations and non-Gaussianities in Hor̆ava-Lifshitz gravity, arXiv: 0904.4187 [INSPIRE].

[21] E. O Colgain and H. Yavartanoo, Dyonic solution of Hořava-Lifshitz gravity, JHEP 08 (2009) 021 [arXiv: 0904.4357] [INSPIRE].

[22] T.P. Sotiriou, M. Visser and S. Weinfurtner, Phenomenologically viable Lorentz-violating quantum gravity, Phys. Rev. Lett. 102 (2009) 251601 [arXiv:0904.4464] [INSPIRE].

[23] T.P. Sotiriou, M. Visser and S. Weinfurtner, Quantum gravity without Lorentz invariance, JHEP 10 (2009) 033 [arXiv:0905.2798] [INSPIRE].

[24] S. Mukohyama, K. Nakayama, F. Takahashi and S. Yokoyama, Phenomenological aspects of Hor̆ava-Lifshitz cosmology, Phys. Lett. B 679 (2009) 6 [arXiv:0905.0055] [INSPIRE].

[25] Y.S. Myung and Y.-W. Kim, Thermodynamics of Hor̆ava-Lifshitz black holes, Eur. Phys. J. C 68 (2010) 265 [arXiv:0905.0179] [INSPIRE].

[26] R.-G. Cai, L.-M. Cao and N. Ohta, Thermodynamics of black holes in Hor̆ava-Lifshitz gravity, Phys. Lett. B 679 (2009) 504 [arXiv:0905.0751] [inSPIRE].

[27] Y.S. Myung, Thermodynamics of black holes in the deformed Horava-Lifshitz gravity, Phys. Lett. B 678 (2009) 127 [arXiv:0905. 0957] [INSPIRE].

[28] D. Orlando and S. Reffert, On the renormalizability of Horava-Lifshitz-type gravities, Class. Quant. Grav. 26 (2009) 155021 [arXiv:0905.0301] [INSPIRE].

[29] T. Nishioka, Hořava-Lifshitz holography, Class. Quant. Grav. 26 (2009) 242001 [arXiv: 0905.0473] [INSPIRE].

[30] R.A. Konoplya, Towards constraining of the Hořava-Lifshitz gravities, Phys. Lett. B 679 (2009) 499 [arXiv: 0905. 1523] [INSPIRE].

[31] S.-B. Chen and J.-L. Jing, Strong field gravitational lensing in the deformed Horava-Lifshitz black hole, Phys. Rev. D 80 (2009) 024036 [arXiv:0905.2055] [INSPIRE]. 
[32] B. Chen, S. Pi and J.-Z. Tang, Scale invariant power spectrum in Hor̆ava-Lifshitz cosmology without matter, JCAP 08 (2009) 007 [arXiv:0905.2300] [INSPIRE].

[33] C. Charmousis, G. Niz, A. Padilla and P.M. Saffin, Strong coupling in Hor̆ava gravity, JHEP 08 (2009) 070 [arXiv: 0905. 2579] [inSPIRE].

[34] M. Li and Y. Pang, A trouble with Hor̆ava-Lifshitz gravity, JHEP 08 (2009) 015 [arXiv: 0905.2751] [INSPIRE].

[35] Y.-W. Kim, H.W. Lee and Y.S. Myung, Nonpropagation of scalar in the deformed Horaava-Lifshitz gravity, Phys. Lett. B 682 (2009) 246 [arXiv:0905.3423] [INSPIRE].

[36] E.N. Saridakis, Hořava-Lifshitz dark energy, Eur. Phys. J. C 67 (2010) 229 [arXiv:0905.3532] [INSPIRE].

[37] S. Mukohyama, Dark matter as integration constant in Hor̆ava-Lifshitz gravity, Phys. Rev. D 80 (2009) 064005 [arXiv: 0905.3563] [INSPIRE].

[38] G. Calcagni, Detailed balance in Hořava-Lifshitz gravity, Phys. Rev. D 81 (2010) 044006 [arXiv:0905.3740] [INSPIRE].

[39] M. Botta-Cantcheff, N. Grandi and M. Sturla, Wormhole solutions to Hor̆ava gravity, Phys. Rev. D 82 (2010) 124034 [arXiv: 0906.0582] [INSPIRE].

[40] J. Bellorín, A. Restuccia and A. Sotomayor, Wormholes in Hořava gravity with cosmological constant, arXiv: 1501.04568 [INSPIRE].

[41] J. Bellorín, A. Restuccia and A. Sotomayor, Wormholes and naked singularities in the complete Hořava theory, Phys. Rev. D 90 (2014) 044009 [arXiv: 1404.2884] [INSPIRE].

[42] E.B. Kiritsis and G. Kofinas, On Hořava-Lifshitz 'black holes', JHEP 01 (2010) 122 [arXiv: 0910.5487] [INSPIRE].

[43] R.-G. Cai and N. Ohta, Horizon thermodynamics and gravitational field equations in Hor̆ava-Lifshitz gravity, Phys. Rev. D 81 (2010) 084061 [arXiv:0910.2307] [INSPIRE].

[44] P. Hořava and C.M. Melby-Thompson, General covariance in quantum gravity at a Lifshitz point, Phys. Rev. D 82 (2010) 064027 [arXiv:1007.2410] [INSPIRE].

[45] S. Mukohyama, Hořava-Lifshitz cosmology: a review, Class. Quant. Grav. 27 (2010) 223101 [arXiv: 1007.5199] [INSPIRE].

[46] A.E. Gumrukcuoglu, S. Mukohyama and A. Wang, General relativity limit of Horava-Lifshitz gravity with a scalar field in gradient expansion, Phys. Rev. D 85 (2012) 064042 [arXiv: 1109.2609] [INSPIRE].

[47] K. Lin, S. Mukohyama and A. Wang, Solar system tests and interpretation of gauge field and Newtonian prepotential in general covariant Hořava-Lifshitz gravity, Phys. Rev. D 86 (2012) 104024 [arXiv: 1206.1338 ] [INSPIRE].

[48] M. Bañados, C. Teitelboim and J. Zanelli, The black hole in three-dimensional space-time, Phys. Rev. Lett. 69 (1992) 1849 [hep-th/9204099] [INSPIRE].

[49] R.B. Mann, J.J. Oh and M.-I. Park, The role of angular momentum and cosmic censorship in the (2+1)-dimensional rotating shell collapse, Phys. Rev. D 79 (2009) 064005 [arXiv:0812.2297] [INSPIRE].

[50] M.-I. Park, Smeared hair and black holes in three-dimensional de Sitter spacetime, Phys. Rev. D 80 (2009) 084026 [arXiv:0811.2685] [INSPIRE]. 
[51] J.P.S. Lemos, Cylindrical black hole in general relativity, Phys. Lett. B 353 (1995) 46 [gr-qc/9404041] [INSPIRE].

[52] Y. Lee, G. Kang, H.-C. Kim and J. Lee, String or brane-like solutions in four-dimensional Einstein gravity in the presence of cosmological constant, Phys. Rev. D 84 (2011) 084042 [arXiv: 1108.3031] [INSPIRE].

[53] S. Aminneborg, I. Bengtsson, S. Holst and P. Peldan, Making anti-de Sitter black holes, Class. Quant. Grav. 13 (1996) 2707 [gr-qc/9604005] [INSPIRE].

[54] R.B. Mann, Pair production of topological anti-de Sitter black holes, Class. Quant. Grav. 14 (1997) L109 [gr-qc/9607071] [inSPIRE].

[55] R.-G. Cai and Y.-Z. Zhang, Black plane solutions in four-dimensional space-times, Phys. Rev. D 54 (1996) 4891 [gr-qc/9609065] [INSPIRE].

[56] L. Vanzo, Black holes with unusual topology, Phys. Rev. D 56 (1997) 6475 [gr-qc/9705004] [INSPIRE].

[57] D.R. Brill, J. Louko and P. Peldan, Thermodynamics of $(3+1)$-dimensional black holes with toroidal or higher genus horizons, Phys. Rev. D 56 (1997) 3600 [gr-qc/9705012] [INSPIRE].

[58] R. Penrose, Gravitational collapse and space-time singularities, Phys. Rev. Lett. 14 (1965) 57 [INSPIRE].

[59] R.B. Mann, Black holes of negative mass, Class. Quant. Grav. 14 (1997) 2927 [gr-qc/9705007] [INSPIRE].

[60] Supernova Search Team collaboration, A.G. Riess et al., Type Ia supernova discoveries at $z>1$ from the Hubble Space Telescope: evidence for past deceleration and constraints on dark energy evolution, Astrophys. J. 607 (2004) 665 [astro-ph/0402512] [INSPIRE].

[61] B. Ryden, Introduction to cosmology, Addison-Wesley, San Francisco U.S.A. (2003).

[62] C. Argüelles, N. Grandi and M.-I. Park, Formal aspects of black holes in Hořava-Lifshitz gravity, work in progress.

[63] M.-I. Park, The rotating black hole in renormalizable quantum gravity: the three-dimensional Hořava gravity case, Phys. Lett. B 718 (2013) 1137 [arXiv:1207.4073] [INSPIRE].

[64] Y.-B. Kim, C.Y. Oh and N. Park, Classical geometry of de Sitter space-time: an introductory review, hep-th/0212326 [INSPIRE].

[65] M. Minamitsuji, Classification of cosmology with arbitrary matter in the Horava-Lifshitz theory, Phys. Lett. B 684 (2010) 194 [arXiv:0905.3892] [INSPIRE].

[66] A. Wang and Y. Wu, Thermodynamics and classification of cosmological models in the Horava-Lifshitz theory of gravity, JCAP 07 (2009) 012 [arXiv:0905.4117] [INSPIRE].

[67] K.-I. Maeda, Y. Misonoh and T. Kobayashi, Oscillating universe in Horava-Lifshitz gravity, Phys. Rev. D 82 (2010) 064024 [arXiv:1006.2739] [InSPIRE].

[68] I. Cho and G. Kang, Four dimensional string solutions in Hor̆ava-Lifshitz gravity, JHEP 07 (2010) 034 [arXiv:0909.3065] [INSPIRE].

[69] A.N. Aliev and C. Senturk, Black strings in Horava-Lifshitz gravity, Phys. Rev. D 84 (2011) 044010 [arXiv: 1106.0024$]$ [INSPIRE].

[70] K. Skenderis and P.K. Townsend, Pseudo-supersymmetry and the domain-wall/cosmology correspondence, J. Phys. A 40 (2007) 6733 [hep-th/0610253] [INSPIRE]. 